\title{
Gauge theories for the thermal Hall effect
}

\author{
Haoyu Guo, Rhine Samajdar, Mathias S. Scheurer, and Subir Sachdev \\ Department of Physics, Harvard University, Cambridge MA 02138, USA
}

(Dated: April 23, 2020)

\begin{abstract}
We consider the thermal Hall effect of fermionic matter coupled to emergent gauge fields in $2+1$ dimensions. While the low-temperature thermal Hall conductivity of bulk topological phases can be connected to chiral edge states and a gravitational anomaly, there is no such interpretation at nonzero temperatures above $2+1$ dimensional quantum critical points. In the limit of a large number of matter flavors, the leading contribution to the thermal Hall conductivity is that from the fermionic matter. The next-to-leading contribution is from the gauge fluctuations, and this has a sign which is opposite to that of the matter contribution. We illustrate this by computations on a Dirac ChernSimons theory of the quantum phase transition in a square-lattice antiferromagnet involving the onset of semion topological order. We find similar results for a model of the pseudogap metal with Fermi pockets coupled to an emergent U(1) gauge field. We note connections to recent observations on the hole-doped cuprates: our theory captures the main trends, but the overall magnitude of the effect is smaller than that observed.
\end{abstract}

\section{CONTENTS}

I. Introduction

A. Undoped insulator

B. Pseudogap at nonzero doping

II. Free Dirac fermion

A. Transport contribution from the Kubo formula

B. Internal magnetization

III. Maxwell-Chern-Simons Theory
A. Propagator
B. Stress-tensor vertex
C. Stress tensor-stress tensor correlation function
D. $T=0$ : Gravitational Chern-Simons term
E. Finite $T$ : Thermal Hall Effect

1

2

2

3

4

4

IV. Dirac Chern-Simons Theory

A. Gauge fluctuations in the $N_{f} \rightarrow \infty$ limit

B. Gauge fluctuations at next-to-leading order

V. Conclusion

Acknowledgments

A. Low-energy field theory

B. Derivation of heat current and the associated Feynman rules

1. Equation-of-motion approach

2. Noether procedure

C. Gluon propagator

1. Limit of zero external momentum
D. Framing anomaly in the 'wrong' metric

References

\section{INTRODUCTION}

Recent experiments have shown that the thermal Hall effect, also known as the the Righi-Leduc effect, is a powerful probe for the presence of unconventional excitations in correlated electron systems. For instance, 6 in the spin liquid candidate $\alpha-\mathrm{RuCl}_{3}$, the temperature 6 and field dependence of the thermal Hall coefficient, $\kappa_{x y}$, 6 has been suggested to indicate the presence of neutral excitations with exotic statistics [1].

Grissonnanche et al. [2] measured the thermal Hall effect in the normal state of four different copper-based superconductors. In the overdoped compounds, they observed a conventional $\kappa_{x y}$, related to the electrical 9 Hall conductivity, $\sigma_{x y}$, by the Wiedemann-Franz law. 10 Interestingly, with decreasing doping, they observed the onset of a negative contribution to $\kappa_{x y}$ upon entering the pseudogap phase, which is unrelated to $\sigma_{x y}$. This negative signal increases in magnitude with lowering doping, and persists all the way into the insulator. Given that a much smaller response is expected from conventional spin-wave theory [3, 4], Grissonnanche et al. argued that it indicated the presence of exotic neutral excitations in the pseudogap phase. In the present work, we employ a gauge theory for the pseudogap phase [5-9], and propose that the emergent gauge field is a neutral excitation which could help produce the observed $\kappa_{x y}$.

In a previous study [10], we focused on the thermal Hall effect in the insulator, and illustrated that the proximity to a quantum phase transition-between the Néel state and a state with coexisting Néel and semion topo- 
logical order - could explain the enhanced thermal Hall effect in the insulator. This enhanced $\kappa_{x y}$ was computed using the gauge theory for the critical point, which had four different formulations, all dual to each other. As the gauge theory is strongly coupled, the computation relied on an expansion in $1 / N_{f}$, where $N_{f}$ is the number of flavors of matter fields. The calculation of $\kappa_{x y}$ at $N_{f}=\infty$ was described in Ref. 10, and we will present further details here. We will also describe the structure of the leading $1 / N_{f}$ corrections to $\kappa_{x y}$; we argue that an important component of these corrections (resulting from the analog of the 'Aslamazov-Larkin' diagrams) can be interpreted as the contribution of the collective mode associated with the emergent gauge field to $\kappa_{x y}$. This interpretation will be useful to us when we turn to consideration of the doped case in the latter part of this paper.

We now outline the models studied and the main results for the undoped and doped cases in turn.

\section{A. Undoped insulator}

We focus our attention on one of the four dualityequivalent gauge theories describing the vicinity of the onset of semion topological order in the Néel state [10]: the $\mathrm{SU}(2)$ gauge theory at Chern-Simons level $k=-1 / 2$, coupled to a single flavor $\left(N_{f}=1\right)$ of a two-component Dirac fermion $\Psi$ with mass $m$. We generalize the fermions to $\Psi_{\ell}$ with $\ell=1 \ldots N_{f}$ flavors, and consider the Lagrangian

$$
\mathcal{L}_{\Psi}=\bar{\Psi}_{\ell}\left[i \gamma^{\mu}\left(\partial_{\mu}-i A_{\mu}\right)\right] \Psi_{\ell}+m \bar{\Psi}_{\ell} \Psi_{\ell}+k C S\left[A_{\mu}\right],
$$

where $A_{\mu}$ is the $\mathrm{SU}(2)$ gauge field, $k$ is the Chern-Simons level, and $\Psi$ is a continuum field derived from the lattice model in Appendix A; $\bar{\Psi}=\Psi^{\dagger} \gamma^{0}$. For $N_{f}=1$ and $k=$ $-1 / 2$, the $m>0$ phase of (1.1) is 'trivial' and describes the conventional Néel state, while the $m<0$ phase of (1.1) has semion topological order [10]. The derivation of this field theory starting from a microscopic lattice model is sketched in Appendix A.

The thermal Hall conductivity of (1.1) is expected to obey

$$
\kappa_{x y}=\frac{k_{B}^{2} T}{\hbar} \mathcal{K}(m / T)
$$

where $\mathcal{K}$ is a dimensionless universal function of $m / T$, with $m$ the renormalized mass of the lowest quasiparticle excitations and $T$ the absolute temperature. In the limit $|m| / T \rightarrow \infty$, the exact values of $\kappa_{x y}$ can be deduced from arguments based on gravitational anoma- lies as [10-12]

$$
\kappa_{x y}=\frac{\pi k_{B}^{2} T}{6 \hbar} \operatorname{sgn}(\hat{k})\left[2|\hat{k}|-\frac{3|\hat{k}|}{|\hat{k}|+2}\right] ; \quad \frac{|m|}{T} \rightarrow \infty,
$$

where the integer $\hat{k}$ is defined by

$$
\hat{k}=k+\frac{N_{f}}{2} \operatorname{sgn}(m)
$$

and the sign function vanishes for zero argument, i.e., $\operatorname{sgn}(0)=0$. We will obtain the large- $N_{f}$ limit of the result (1.3) below in a direct $1 / N_{f}$ expansion, with $k$ taken to be of order $N_{f}$. Note that the first term in Eq. (1.3) is of order $N_{f}^{1}$, while the second term is of order $N_{f}^{0}$.

Our $1 / N_{f}$ expansion also yields a simple interpretation of the two terms in (1.3). The leading term of order $N_{f}$ is the contribution of free Dirac fermions, where we assume that the Chern-Simons term in $A_{\mu}$ was generated by integrating out massive Dirac fermions. The contribution to the universal scaling function $\mathcal{K}$ by the free Dirac fermions is specified by (2.11) and plotted in Fig. 2. The subleading term of order $N_{f}^{0}$ is the contribution of the fluctuations of $A_{\mu}$. We will exploit this interpretation when we consider the doped case.

We also consider the quantum critical limit, $|m| / T \rightarrow$ 0 , when neither an exact computation of $\kappa_{x y}$ is possible, and nor is $\kappa_{x y}\left(\hbar /\left(\pi k_{B}^{2}\right)\right)$ expected to be quantized at a rational value. In this case, we obtain

$$
\kappa_{x y}=\frac{\pi k_{B}^{2} T}{6 \hbar}\left[2 k+\mathcal{O}\left(N_{f}^{0}\right)\right] \quad, \quad \frac{|m|}{T} \rightarrow 0 .
$$

The computation of the $\mathcal{O}\left(N_{f}^{0}\right)$ number requires a lengthy numerical computation which we will outline, but not carry out to completion. We note that we do not expect $\kappa_{x y}$ in the limit $|m| / T \rightarrow 0$ to be related to any gravitational anomaly or contact terms $[13,14]$; the latter are evaluated at $T=0$, and not in the limit required for a quantum critical transport coefficient, with frequencies much smaller than $T[15,16]$.

\section{B. Pseudogap at nonzero doping}

We will describe the pseudogap by essentially the same theory as that used in Ref. 6, which was successfully compared with numerical studies of the Hubbard model $[6,7,9]$ and photoemission experiments on an electron-doped cuprate [17]. In the limit of the insulating state, and in the vicinity of the onset of semion topological order in the presence of Néel order as discussed in Section I A, this theory can be related [10] to one of the theories which are equivalent to (1.1) after 
duality - a SU(2) gauge theory at Chern-Simons level 1, coupled to a complex scalar which is a $\mathrm{SU}(2)$ fundamental. While the fermionic $\mathrm{SU}(2)$ theory at level $-1 / 2$ in (1.1) was useful in describing $\kappa_{x y}$ in the insulator [10], the complex scalar $\mathrm{SU}(2)$ theory is far more convenient in the doped case. This is because the latter theory has fermionic charge carriers, and this allows easy access to a metallic state at nonzero doping.

The pseudogap metal is described by transforming to a rotating reference frame in spin space [5], which results in a $\mathrm{SU}(2)$ gauge theory. The fluctuating spin density wave order acts like a Higgs field, which breaks the $\mathrm{SU}(2)$ invariance down to $\mathrm{U}(1)$. Coupled to the $\mathrm{U}(1)$ gauge field, $a_{\mu}$, we have bosonic spinons and fermionic chargons $f_{p}$ with $\mathrm{U}(1)$ gauge charges $p= \pm 1$. We focus on the fermionic chargons, as they form Fermi pockets with charged gapless excitations on the Fermi surface. We write down a simple effective theory for these chargons $[6,7,18]$ :

$$
\begin{aligned}
\mathcal{L}_{f}= & \sum_{v=1,2} \sum_{p= \pm 1} f_{p v}^{\dagger}\left(\frac{\partial}{\partial \tau}-\mu-i p a_{\tau}\right. \\
& \left.-\frac{\left(\boldsymbol{\nabla}-i p \boldsymbol{a}-i e \boldsymbol{A}_{\mathrm{em}}\right)^{2}}{2 m^{*}}+v_{\mathrm{dis}}(\boldsymbol{r})\right) f_{p v}
\end{aligned}
$$

Here, $v$ is a valley index, $m^{*}$ is the effective mass of the fermions (we have ignored mass anisotropies), $\mu$ is a chemical potential, $a_{\mu}=\left(a_{\tau}, \vec{a}\right)$ is the emergent $\mathrm{U}(1)$ gauge field, and $\boldsymbol{A}_{\mathrm{em}}$ is the fixed background electromagnetic gauge field associated with the applied magnetic field $B=\hat{\boldsymbol{z}} \cdot\left(\boldsymbol{\nabla} \times \boldsymbol{A}_{\mathrm{em}}\right)$. We have included a disorder potential $v_{\text {dis }}(\boldsymbol{r})$, because we will consider Hall transport in the weak-field regime $\omega_{c} \tau \ll 1$, where $\omega_{c}$ is the cyclotron frequency and $\tau$ is the elastic scattering time associated with the disorder.

First, let us ignore the internal gauge field $a_{\mu}$. Then, the $f$ fermions form a conventional Fermi liquid, and for $\omega_{c} \tau \ll 1$, the electrical and thermal Hall responses are given by familiar expressions involving the WiedemannFranz relation

$$
\rho_{x y}=\frac{B}{n e c}, \quad \sigma_{x y} \simeq \frac{\rho_{x y}}{\rho_{x x}^{2}}, \quad \kappa_{x y}^{0}=\frac{\pi^{2} T}{3}\left(\frac{k_{B}}{e}\right)^{2} \sigma_{x y},
$$

where $n$ is the total density of the $f$ fermions. Now, let us consider the contribution of $a_{\mu}$ to the thermal Hall response. We will compute this by a simple MaxwellChern-Simons action for $a_{\mu}$

$\mathcal{L}_{a}=\frac{K_{1}}{2}(\boldsymbol{\nabla} \times \boldsymbol{a})^{2}+\frac{K_{2}}{2}\left(\boldsymbol{\nabla} a_{\tau}-\partial_{\tau} \boldsymbol{a}\right)^{2}-\frac{i \sigma_{x y}}{2 e^{2}} \epsilon_{\mu \nu \lambda} a_{\mu} \partial_{\nu} a_{\lambda}$.

We assume that the predominant contribution to the Maxwell terms arises from integrating out the gapped spinons. Integrating out the fermionic chargons introduces the Chern-Simons term in (1.8), proportional to the Hall conductivity of the fermions in (1.7); such a term is also permitted under the symmetry constraints on this gauge theory of doped antiferromagnets $[19,20]$. In general, because of the presence of disorder, the couplings $K_{1,2}$ will also be functions of spatial position; we replace them by their spatial average, and do not expect fluctuations to significantly modify the results presented here. The fermions also introduce singular terms in the transverse gauge field propagator arising from Landau damping [21], so that a more complete effective action is

$$
\mathcal{S}_{a}=\int d^{2} x d \tau \mathcal{L}_{a}+\int \frac{d^{2} k d \omega}{8 \pi^{3}} \gamma_{k}|\omega|\left[\boldsymbol{a}^{T}(k, \omega)\right]^{2},
$$

where $\gamma_{k} \sim 1 / k$ for $k v_{F} \tau \gg 1$, and $\gamma_{k} \sim$ constant for $k v_{F} \tau \ll 1$. Although the term in (1.9) could make a significant contribution to the thermal Hall effect, we leave an analysis of its effects to future work.

Computing the thermal Hall response of the MaxwellChern-Simons theory $\mathcal{L}_{a}$ in Section III, we find that it yields a correction $\kappa_{x y}^{1}$, which has the opposite sign from $\kappa_{x y}^{0}$ in (1.7). This sign change is similar to that in (1.3) between the $\mathcal{O}\left(N_{f}\right)$ term (from the fermions) and the $\mathcal{O}\left(N_{f}^{0}\right)$ term (from the gauge field). The universal function $\mathcal{K}$ in (1.2) for the Maxwell-Chern-Simons theory is specified in (3.29) as a function of the 'topological mass' $m_{t}=\sigma_{x y} /\left(e^{2} K_{2}\right)$, and is plotted in Fig. 4. Note that the universal function (2.11) for Dirac fermions in Fig. 2 does not reduce to the gauge-field function in (3.29) and Fig. 4 by a rescaling of axes: this is evidence that the $T>0$ thermal Hall conductivity is a bulk property, and is not specified by any topological field theory or gravitational anomaly.

We begin our analysis by describing the thermal Hall response of two free theories: a free Dirac fermion in Section II, and free Maxwell-Chern-Simons theory in Section III. The results of the Maxwell-Chern-Simons theory apply directly to the effective theory for the doped pseudogap phase in Eq. (1.8). We will combine Sections II and III to obtain results for the Dirac Chern-Simons theory (1.1) in the $1 / N_{f}$ expansion in Section IV.

\section{FREE DIRAC FERMION}

In order to obtain a finite thermal Hall effect, timereversal symmetry must be broken: this can be achieved by either an external magnetic field or intrinsic magnetic ordering [22]. However, initial attempts to calculate this response based on direct application of the Kubo formula were found to suffer from unphysical divergences 
at zero temperature $[23,24]$. This is because in a system breaking time-reversal symmetry, a temperature gradient drives not only the transport (heat) current, but also an experimentally unobservable circulating current $[25,26]$. Both contributions are present in the microscopic current density calculated by the standard linear response theory, necessitating a proper subtraction of the circulating component. Ref. 27 showed that the electromagnetic and gravitomagnetic energy magnetizations [28, 29] naturally emerge as corrections to the thermal transport coefficients, removing the aforementioned divergences in the process. A subtlety pointed out in Ref. 30 is that the energy magnetization and the thermal Hall coefficient are relative: only the difference between two systems are physically meaningful. We choose to normalize $\kappa_{x y}$ such that the $\kappa_{x y} / T \rightarrow 0$ as $m / T \rightarrow 0$, i.e., the vacuum has zero thermal Hall coefficient.

We now present details of the computation of the thermal Hall coefficient of a free Dirac fermion with a mass $m$ which can be scanned through zero at $T>0$. This is the theory (1.1) without the gauge field $A_{\mu}$. While we consider a single two-component Dirac fermion, note that, because of the $\mathrm{SU}(2)$ gauge index, the theory (1.1) has $2 N_{f}$ such fermions. In the following, we determine both the Kubo part and the magnetization separately, identifying precisely what the transport currents and the magnetizations are, and illustrating how they can be evaluated for a general continuum theory.

\section{A. Transport contribution from the Kubo formula}

The leading contribution to $\kappa_{x y}$ is given by a single fermion polarization bubble shown in Fig. 1.
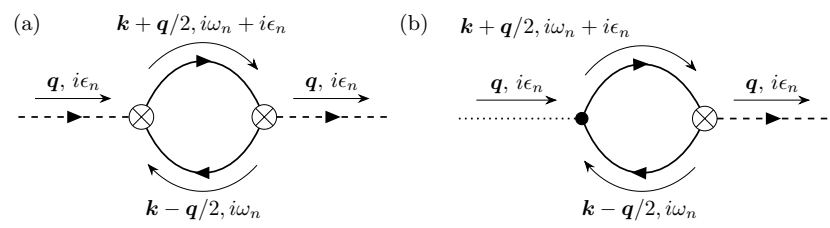

FIG. 1. The fermion polarization bubbles that give the mean-field Kubo (left) and internal magnetization (right) contributions to the thermal Hall conductivity; the crossed circles represent thermal current vertices.

Summing over the internal momentum $\boldsymbol{k}$ and Matsubara frequency $i \omega_{n}$, this diagram evaluates to

$$
\Pi_{x y}^{\mathrm{Q}}\left(\boldsymbol{q}, i \epsilon_{n}\right)=\frac{1}{\beta V} \sum_{\boldsymbol{k}, i \omega_{n}} \operatorname{Tr}\left[\mathcal{V}_{x}^{\mathrm{Q}}\left(-\boldsymbol{q},-i \epsilon_{n}\right)\right.
$$

$$
\left.\mathcal{G}\left(\boldsymbol{k}-\boldsymbol{q} / 2, i \omega_{n}\right) \mathcal{V}_{y}^{\mathrm{Q}}\left(\boldsymbol{q}, i \epsilon_{n}\right) \mathcal{G}\left(\boldsymbol{k}+\boldsymbol{q} / 2, i \omega_{n}+i \epsilon_{n}\right)\right]
$$

where $G_{\Psi}\left(\boldsymbol{k}, i \omega_{n}\right)=1 /\left(-i \omega_{n}+\boldsymbol{\sigma} \cdot \boldsymbol{k}+m \sigma^{z}\right)$ is the free fermion Green's function, and $\mathcal{V}_{a}^{\mathrm{Q}}\left(\boldsymbol{q}, i \epsilon_{n}\right)=\sigma^{a}\left(i \omega_{n}+\right.$ $\left.i \epsilon_{n} / 2\right)$ is the heat/energy-current vertex, derived in Appendix B. The response function is defined as

$$
\begin{aligned}
L_{x y} & =\frac{1}{\epsilon_{n}} \Pi_{x y}^{\mathrm{Q}}\left(\boldsymbol{q}, i \epsilon_{n}\right) \\
& =\frac{T}{2 \epsilon_{n} V} \sum_{\boldsymbol{k}, i \omega_{n}} \frac{-\left(\epsilon_{n}+2 \omega_{n}\right)^{2}}{k_{x}^{2}+k_{y}^{2}+m^{2}+\omega_{n}^{2}} \\
& \times \frac{2 k_{x} k_{y}+m \epsilon_{n}}{k_{x}^{2}+k_{y}^{2}+m^{2}+\left(\epsilon_{n}+\omega_{n}\right)^{2}} \text { as } \boldsymbol{q} \rightarrow 0,
\end{aligned}
$$

specializing to the case of zero external momentum. The numerator of the polarization tensor $\Pi_{\mu \nu}^{\mathrm{Q}}$ consists of a part proportional to $k_{\mu} k_{\nu}$ and a term $\sim \delta_{\mu \nu}$; we can drop the former because it is odd in $k_{x}$ and $k_{y}$ and hence, vanishes upon integration over all momenta. Performing the Matsubara summation and converting the momentum sum to an integral, we get

$$
L_{x y}=-\frac{1}{\epsilon_{n}} \int \frac{\mathrm{d}^{2} \boldsymbol{k}}{(2 \pi)^{2}} \frac{m \epsilon_{n}}{2 \xi_{\boldsymbol{k}}} \tanh \left(\frac{\beta \xi_{\boldsymbol{k}}}{2}\right),
$$

where $\xi_{\boldsymbol{k}}=\sqrt{k_{x}^{2}+k_{y}^{2}+m^{2}}$. Finally, introducing the shorthand $u=\beta \xi_{\boldsymbol{k}}$, we have

$$
\begin{aligned}
L_{x y} & =-\int_{\beta|m|}^{\infty} \frac{\mathrm{d} u}{4 \pi \beta} m \tanh \left(\frac{u}{2}\right) \\
& =-\left.\frac{m}{2 \pi \beta} \ln \left[\cosh \left(\frac{u}{2}\right)\right]\right|_{\beta|m|} ^{\infty} .
\end{aligned}
$$

A few comments are in order about this result. First, we have regulated the integral by introducing a UV cutoff $\Lambda$ but, as we shall see, this drops out eventually. Further, to obtain the DC response, we need to analytically continue to real frequencies $i \epsilon_{n} \rightarrow \epsilon+i 0^{+}$, and then take the limit $\epsilon \rightarrow 0$ after $\boldsymbol{q} \rightarrow 0$. The thermal Hall coefficient is then $[27,31]$

$$
\begin{aligned}
\kappa_{x y}^{\text {Kubo }} & \equiv \frac{L_{x y}}{T} \\
& =\frac{m}{2 \pi}\left\{\ln \left[\cosh \left(\frac{\beta|m|}{2}\right)\right]-\ln \left[\cosh \left(\frac{\beta \Lambda}{2}\right)\right]\right\} .
\end{aligned}
$$

\section{B. Internal magnetization}

The second contribution to the conductivity comes from the circulating heat current. The zero-field heat 
magnetization can be calculated from the differential equation [27]

$$
2 \boldsymbol{M}_{Q}-T \frac{\partial \boldsymbol{M}_{Q}}{\partial T}=\frac{1}{2 i} \boldsymbol{\nabla}_{\boldsymbol{q}} \times\left.\left\langle\hat{K}_{-\boldsymbol{q}} ; \hat{J}_{\boldsymbol{q}}^{Q}\right\rangle_{0}\right|_{\boldsymbol{q} \rightarrow 0},
$$

where $\hat{K}_{\boldsymbol{q}}$ is the Fourier transform of $\hat{K}(\boldsymbol{r})=h(\boldsymbol{r})-$ $\mu \hat{n}(\boldsymbol{r}), h$ and $\hat{n}$ being the local energy and number densities, respectively. The correlator on the RHS is evaluated in the static limit, i.e. $\boldsymbol{q} \rightarrow 0$ after $\epsilon \rightarrow 0$. We also point out to readers that Eq. (2.5), cited from Ref. 27, only applies to systems whose energy current depends on the gravitational field in a particular way, while the general formalism is discussed in Ref. 30.

Equipped with the structure of this modified vertex from Appendix B, we now evaluate Eq. (2.5) piece by piece. Consider the first term in the curl; retaining only the terms even in the internal momentum [32], we get

$$
\begin{aligned}
& \partial_{q_{x}}\left\langle\hat{K}_{-\boldsymbol{q}} ; \hat{J}_{y, \boldsymbol{q}}^{Q}\right\rangle \\
& =\partial_{q_{x}}\left(\frac{1}{\beta V} \sum_{\boldsymbol{k}, i \omega_{n}} \frac{2 i m q_{x}\left(\epsilon_{n}+2 \omega_{n}\right)^{2}}{4\left(|\boldsymbol{k}-\boldsymbol{q} / 2|^{2}+m^{2}+\omega_{n}^{2}\right)}\right. \\
& \left.\quad \quad \times \frac{1}{\left(|\boldsymbol{k}+\boldsymbol{q} / 2|^{2}+m^{2}+\left(\epsilon_{n}+\omega_{n}\right)^{2}\right)}\right)\left.\right|_{\epsilon_{n}=0} \\
& =i \int \frac{\mathrm{d}^{2} \boldsymbol{k}}{(2 \pi)^{2}} \frac{m\left(\beta \xi_{\boldsymbol{k}}+\sinh \left(\beta \xi_{\boldsymbol{k}}\right)\right) \operatorname{sech}^{2}\left(\beta \xi_{\boldsymbol{k}} / 2\right)}{4 \xi_{\boldsymbol{k}}} \\
& =i \int_{\beta|m|}^{\infty} \frac{\mathrm{d} u}{8 \pi \beta} m u(u+\sinh (u)) \operatorname{sech}^{2}(u / 2) \\
& =\left.\frac{i m u}{4 \pi \beta} \tanh \left(\frac{u}{2}\right)\right|_{\beta|m|} ^{\infty} .
\end{aligned}
$$

In the second line, we evaluate the sum at $\epsilon_{n}=0$ and then take the $q_{x}$ derivative. Similarly, as expected by symmetry,

$$
\partial_{q_{y}}\left\langle\hat{K}_{-\boldsymbol{q}} ; \hat{J}_{x, \boldsymbol{q}}^{Q}\right\rangle=-\left.\frac{i m u}{4 \pi \beta} \tanh \left(\frac{u}{2}\right)\right|_{\beta|m|} ^{\infty} .
$$

Plugging Eqs. (2.6) and (2.7) back into Eq. (2.5), we have

$$
\begin{aligned}
& 2 \boldsymbol{M}_{Q}-T \frac{\partial \boldsymbol{M}_{Q}}{\partial T}= \\
& \frac{m}{4 \pi}\left[\Lambda \tanh \left(\frac{\beta \Lambda}{2}\right)-|m| \tanh \left(\frac{\beta|m|}{2}\right)\right],
\end{aligned}
$$

which can be solved for $\boldsymbol{M}_{Q}$ to obtain

$$
M_{Q}=c_{1} T^{2}-\frac{m T}{4 \pi}\left[\frac{2 \mathrm{Li}_{2}\left(-e^{-\Lambda / T}\right)}{\Lambda / T}-\frac{2 \mathrm{Li}_{2}\left(-e^{-|m| / T}\right)}{|m| / T}\right.
$$

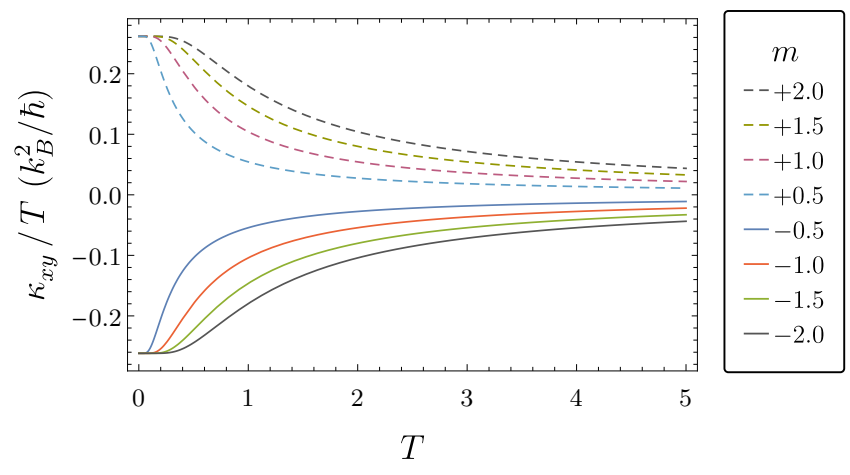

FIG. 2. The thermal Hall conductivity of a free Dirac fermion of mass $m$. The values as $T \rightarrow 0$ are $\pm \pi / 12$.

$$
\left.+\frac{|m|-\Lambda}{2 T}+2 \ln \left(\frac{e^{-|m| / T}+1}{e^{-\Lambda / T}+1}\right)\right],
$$

where $c_{1}$ is an arbitrary constant. Now, $\kappa_{x y}^{\mathrm{tr}}=\kappa_{x y}^{\mathrm{Kubo}}+$ $2 M_{Q} / T$. Collecting the terms proportional to $\Lambda$, we get

$$
\begin{aligned}
\lim _{\Lambda \rightarrow \infty} \frac{m}{4 \pi}[ & -2 \ln \left(\cosh \left(\frac{\beta \Lambda}{2}\right)\right)+\beta \Lambda \\
& \left.-\frac{4 \operatorname{Li}_{2}\left(-e^{-\beta \Lambda}\right)}{\beta \Lambda}+4 \ln \left(e^{-\beta \Lambda}+1\right)\right]=\frac{m}{2 \pi} \ln 2,
\end{aligned}
$$

the first two terms cancel out the UV divergences and all dependencies on the cutoff $\Lambda$ drop out. Thus, the physical thermal Hall conductivity is given by

$\kappa_{x y}^{\mathrm{tr}}=2 c_{1} T+\frac{m}{2 \pi}\left[\frac{2 \operatorname{Li}_{2}\left(-e^{-|m| / T}\right)}{|m| / T}-\ln \left(e^{-|m| / T}+1\right)\right]$,

where the first part comes from the magnetization and the last piece is the Kubo contribution.

At this point, the constant $c_{1}$ arising from the solution of the differential equation can be determined as follows. We have seen above that $\kappa_{x y} / T$ is a function of the dimensionless variable $|m| / T$ alone. Therefore, taking the limit $m \rightarrow 0$ or equivalently, $T \rightarrow \infty$ (where we know a priori that $\kappa_{x y} / T$ should go to zero), Eq. (2.11) reduces to

$$
\kappa_{x y}^{\mathrm{tr}}=2 c_{1} T+\frac{\operatorname{sign}(m)}{2 \pi}\left(-2 \cdot \frac{\pi^{2}}{12}\right) T \equiv 0
$$

and the last condition implies that $c_{1}=\operatorname{sign}(m) \pi / 24$. As a result, when $T \rightarrow 0$ keeping $m \neq 0$ and fixed, we obtain $\kappa_{x y}^{\mathrm{tr}} / T=\operatorname{sign}(m) \pi / 12$. The dependence of $\kappa_{x y}^{\mathrm{tr}}$ on temperature and mass is shown in Fig. 2. 


\section{MAXWELL-CHERN-SIMONS THEORY}

In this section, we consider the framing anomaly and thermal Hall response of the U(1) Maxwell-ChernSimons theory (1.8). Our discussion will be restricted to the level of an effective theory and we do not attempt to extract the microscopic values of $K_{1}, K_{2}$. As we will see, the effective theory already provides a satisfactory interpolation between the two topological phases.

The Maxwell-Chern-Simons theory has a speed of 'light' $c_{0}=\sqrt{K_{1} / K_{2}}$. Since there is no other velocity scale in the theory, we can set $c_{0}=1$ (see also Appendix D). The MCS theory (1.8) takes the following relativistic form in real time:

$$
\mathcal{L}=\frac{k}{4 \pi} \varepsilon^{\mu \rho \nu} a_{\mu} \partial_{\rho} a_{\nu}-\frac{1}{4 g} f_{\mu \nu} f^{\mu \nu},
$$

where $f_{\mu \nu}=\partial_{\mu} a_{\nu}-\partial_{\nu} a_{\mu}$. Here, the coupling $g=1 / K_{2}$ has dimensions of energy in $2+1 \mathrm{D}$, and $k=2 \pi \sigma_{x y} / e^{2}$ is the Chern-Simons Level. The large- $N_{f}$ limit of fermion flavor implies $k=\mathcal{O}\left(N_{f}\right)$ and $g=\mathcal{O}\left(1 / N_{f}\right)$. If we are interested in the thermal Hall effect of the gapped phases, we should take $g$ to be the largest energy scale and send $g \rightarrow \infty$.

Chern-Simons theory has a gravitational anomaly called the framing anomaly. It is well known that ChernSimons theory is topological and does not couple to spacetime geometry on the classical level. Witten [33] pointed out that at the quantum level, the theory inevitably couples to a metric because of the gauge-fixing procedure. However, this is not adequate for writing down a sensible stress tensor, because any vertex function due to the gauge-fixing procedure is longitudinal and thus, vanishes when contracted with the physical transverse propagator. Here, we will try an alternative method, by considering the Maxwell-Chern-Simons theory. The Maxwell term serves as a UV regulator and it enables us to write down a stress-tensor vertex. According to Witten [33], the gravitational anomaly appears as a gravitational Chern-Simons term

$$
C S_{g}\left[g_{\mu \nu}\right]=\frac{c}{96 \pi} \int \operatorname{tr}\left(\Gamma \wedge \mathrm{d} \Gamma+\frac{2}{3} \Gamma \wedge \Gamma \wedge \Gamma\right),
$$

where $\Gamma$ is the Christoffel symbol associated with the metric $g_{\mu \nu}$. The prefactor $c$ is the (chiral) central charge

$$
c=-\frac{\operatorname{dim}(G) k}{\left(|k|+c_{2}(G)\right)},
$$

where $c_{2}(G)$ is the dual Coxeter number of the gauge group $G$.

In what follows, we perturbatively compute the gravitational anomaly and thermal Hall effect of the above MCS theory in the large- $k\left(k \propto N_{f}\right)$ limit. Following
[34], we calculate the stress tensor-stress tensor correlation function $\Pi^{\mu \nu ; \rho \lambda}(x, t)=-i\left\langle T^{\mu \nu}(x, t) T^{\rho \lambda}(0,0)\right\rangle$ of the MCS theory. We can interpret $\Pi^{\mu \nu ; \rho \lambda}$ as the effective action (up to a minus sign) $S_{\text {eff }}\left[g_{\mu \nu}\right]$ of metric $g_{\mu \nu}$ in a weakly curved background $g_{\mu \nu}=\eta_{\mu \nu}+h_{\mu \nu},\left|h_{\mu \nu}\right| \ll$ $\left|\eta_{\mu \nu}\right|$. We will show that at zero temperature $\Pi^{\mu \nu ; \rho \lambda}$ agrees with the gravitational Chern-Simons term (3.2).

This gravitational anomaly is proportional to the thermal Hall coefficient at the next-leading large- $N_{f}$ order via the relation

$$
\kappa_{x y}=\frac{\pi}{6} c T
$$

It is argued in Ref. 35 that a gravitational ChernSimons term cannot give rise to a thermal Hall effect from the Kubo formula because it contains three derivatives rather than one. In our calculation, we find that the thermal Hall effect actually arises from the finitetemperature part of $\Pi^{\mu \nu ; \rho \lambda}$, which comes from the same diagrams as the gravitational anomaly and contains only one derivative.

At the next-leading large- $N_{f}$ order, our approach works both for Abelian and non-Abelian theories because $c=\operatorname{dim}(G)+\mathcal{O}\left(1 / N_{f}\right)$, and we simply include $\operatorname{dim}(G)$ copies of gauge fields, which are noninteracting at this order.

\section{A. Propagator}

We add a gauge fixing term $\mathcal{L}_{g f}=\left(\partial_{\mu} a^{\mu}\right)^{2} /(2 \xi g)$ and work out the propagator. Some algebra leads to

$$
\begin{aligned}
& S=\int \frac{\mathrm{d}^{3} p}{(2 \pi)^{3}} \frac{1}{2 g} \\
& a_{\mu}(-p)\left(p^{\mu} p^{\nu}-\eta^{\mu \nu} p^{2}+\frac{p^{\mu} p^{\nu}}{\xi}+\frac{k g}{2 \pi} \varepsilon^{\mu \nu \rho} i p_{\rho}\right) a_{\nu}(p)
\end{aligned}
$$

The propagator is thus

$D^{\mu \nu}(p)=\frac{-g}{p^{2}-m_{t}^{2}}\left[P^{\mu \nu}(p)+\frac{m_{t}}{p^{2}} \varepsilon^{\mu \nu \rho} i p_{\rho}\right]+\frac{\xi}{\left(p^{2}\right)^{2}} p^{\mu} p^{\nu}$,

where the topological mass $m_{t}=k g /(2 \pi)$, and $P^{\mu \nu}(p)=$ $\eta^{\mu \nu}-p^{\mu} p^{\nu} / p^{2}$.

\section{B. Stress-tensor vertex}

In this section, we work out the stress-tensor vertex as shown in Fig. 3. The stress tensor is given by the Maxwell term, which is

$$
T^{\mu \nu}=\frac{-1}{g}\left[f^{\mu \rho} f_{\rho}^{\nu}-\frac{1}{4} \eta^{\mu \nu} f_{\alpha \beta} f^{\alpha \beta}\right] .
$$




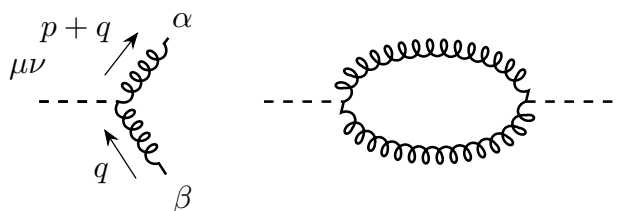

FIG. 3. Left: Vertex diagram of the stress tensor. Right: Diagram of the stress tensor-stress tensor correlation function.
It is worth noticing that the stress tensor above can be derived solely from translation symmetry and gauge invariance, without reference to Lorentz symmetry (see Appendix. B).

We write the vertex function of Fig. 3 as

$$
-\Gamma_{\mu \nu ; \alpha \beta}(p+q, q) / g
$$

where

$$
\begin{aligned}
\Gamma^{\mu \nu ; \alpha \beta}(p+q, q) & =\left[(p+q)^{\mu} q^{\nu}+(p+q)^{\nu} q^{\mu}-\eta^{\mu \nu}(p+q) \cdot q\right] \eta^{\alpha \beta}+(p+q) \cdot q\left(\eta^{\mu \alpha} \eta^{\nu \beta}+\eta^{\mu \beta} \eta^{\nu \alpha}\right) \\
& -\left[q^{\alpha}\left(\eta^{\beta \mu}(p+q)^{\nu}+\eta^{\beta \nu}(p+q)^{\mu}\right)+(p+q)^{\beta}\left(\eta^{\alpha \mu} q^{\nu}+\eta^{\alpha \nu} q^{\mu}\right)-\eta^{\mu \nu} q^{\alpha}(p+q)^{\beta}\right] .
\end{aligned}
$$

\section{Stress tensor-stress tensor correlation function}

We compute the following stress tensor-stress tensor polarization function:

$$
\Pi^{\mu \nu ; \rho \lambda}(x, t)=-i\left\langle T^{\mu \nu}(x, t) T^{\rho \lambda}(0,0)\right\rangle .
$$

In general, the full polarization function should also contain contact terms such as $\left\langle\delta T^{\mu \nu}(x, t) / \delta h_{\rho \lambda}(0,0)\right\rangle$, but those terms are symmetric in $\mu \rho$ and independent of external momentum, so they do not contribute to either the gravitational anomaly or the thermal Hall effect. We only need to consider the single bubble diagram in Fig. 3, which yields

$$
\begin{aligned}
& \Pi^{\mu \nu ; \rho \lambda}(p)=\int \frac{\mathrm{d}^{3} q}{(2 \pi)^{3}} \frac{-i}{2 g^{2}} \Gamma^{\rho \lambda ; \alpha \beta}(p+q, q) i D_{\alpha^{\prime} \alpha}(p+q) \\
& \times \Gamma^{\mu \nu ; \beta^{\prime} \alpha^{\prime}}(q, p+q) i D_{\beta \beta^{\prime}}(q) .
\end{aligned}
$$

Here, we have included a symmetry factor of $1 / 2$.

We want to extract the part which is antisymmetric in $\mu \rho$ and symmetric in $\nu \lambda$, which should ultimately lead to a gravitational Chern-Simons term and thermal Hall effect:

$$
\Pi_{\mathrm{AS}}^{\mu \nu ; \rho \lambda}=\frac{1}{4}\left(\Pi^{\mu \nu ; \rho \lambda}-\Pi^{\rho \nu ; \mu \lambda}+\Pi^{\mu \lambda ; \rho \nu}-\Pi^{\rho \lambda ; \mu \nu}\right) .
$$

\section{D. $T=0$ : Gravitational Chern-Simons term}

At zero temperature, the integrand has Lorentz symmetry, and we can evaluate $\Pi_{\mathrm{AS}}$ using Feynman param- eters:

$$
\begin{aligned}
& \frac{1}{q^{2}(p+q)^{2}\left(q^{2}-m_{t}^{2}\right)\left((p+q)^{2}-m_{t}^{2}\right)}= \\
& 6 \int_{0}^{1} \mathrm{~d} x_{1} \mathrm{~d} x_{2} \mathrm{~d} x_{3} \mathrm{~d} x_{4} \delta\left(\sum x-1\right) \frac{1}{\left(l^{2}-\Delta\right)^{4}}
\end{aligned}
$$

where

$$
\begin{aligned}
l & =q+\left(x_{1}+x_{3}\right) p \\
\Delta & =\left(x_{1}+x_{2}\right) m_{t}^{2}-\left(x_{1}+x_{3}\right)\left(1-x_{1}-x_{3}\right) p^{2}(
\end{aligned}
$$

After some algebra with Mathematica, we have

$$
\begin{aligned}
& \Pi_{\mathrm{AS}}^{\mu \nu ; \rho \lambda}(p)=-\varepsilon^{\mu \rho \sigma} p_{\sigma}\left(\eta^{\nu \lambda} p^{2}-p^{\nu} p^{\lambda}\right) \\
& \times 6 \int_{0}^{1} \mathrm{~d} x_{1} \mathrm{~d} x_{2} \mathrm{~d} x_{3} \mathrm{~d} x_{4} \delta\left(\sum x-1\right)\left(\frac{-m_{t}}{15}\right) \\
& \times \int \frac{\mathrm{d}^{3} l}{(2 \pi)^{3}} \frac{l^{4}\left[10-63\left(x_{1}+x_{3}\right)\left(1-x_{1}-x_{3}\right)\right]+\mathcal{O}\left(l^{2}\right)}{\left(l^{2}-\Delta\right)^{4}} .
\end{aligned}
$$

To obtain the gravitational Chern-Simons term, we isolate the topological contributions by taking the limit $m_{t} \rightarrow \infty$. Note that only the $l^{4}$ term written above can survive the $m_{t} \rightarrow \infty$ limit. The integral can be evaluated using dimensional regularization. The result is

$$
\Pi_{\mathrm{AS}}^{\mu \nu ; \rho \lambda}(p)=\frac{-i}{48 \pi} \operatorname{sgn}\left(m_{t}\right) \varepsilon^{\mu \rho \sigma} p_{\sigma}\left(\eta^{\nu \lambda} p^{2}-p^{\nu} p^{\lambda}\right),
$$

employing the integral formula

$$
\int \frac{\mathrm{d}^{d} l_{E}}{(2 \pi)^{d}} \frac{\left(l_{E}^{2}\right)^{a}}{\left(l_{E}^{2}+D\right)^{b}}=\frac{\Gamma\left(b-a-\frac{d}{2}\right) \Gamma\left(a+\frac{d}{2}\right)}{(4 \pi)^{d / 2} \Gamma(b) \Gamma\left(\frac{d}{2}\right)} \frac{1}{D^{b-a-\frac{d}{2}}} .
$$

We can compare the above result to the gravitational Chern-Simons term (3.2), which, to quadratic order in 
$h$, reduces to

$$
\begin{gathered}
C S_{g}[h]=-\frac{c}{192 \pi} \int \frac{\mathrm{d}^{3} p}{(2 \pi)^{3}} h_{\mu \nu}(-p) \varepsilon^{\mu \rho \sigma}\left(i p_{\sigma}\right) \\
\left(p^{2} \eta^{\nu \lambda}-p^{\nu} p^{\lambda}\right) h_{\rho \lambda}(p) .
\end{gathered}
$$

The correlation function is related to $C S_{g}$ by

$$
\begin{aligned}
\Pi_{\mathrm{AS}}^{\mu \nu ; \rho \lambda}(p) & =-i\left\langle T^{\mu \nu}(p) T^{\rho \lambda}(-p)\right\rangle_{A S} \\
& =-\frac{\delta^{2} C S_{g}[h]}{\delta h_{\mu \nu}(-p) \delta h_{\rho \lambda}(p)}+\text { (symmetrization) } .
\end{aligned}
$$

When evaluating the variation, we get a trivial factor of 2 because $C S_{g}$ is quadratic in $h$. There is another hidden factor of 2 because, when considering variations, we have to include all permutations of $\mu \leftrightarrow \nu, \rho \leftrightarrow \lambda$, which results in four terms. One term is symmetric in $\mu \rho$ and can be dropped. Another term has an apparent $\varepsilon^{\mu \rho \sigma} i p_{\sigma}$ factor. The other two terms are not totally antisymmetric in $\mu \rho$, but after antisymmetrization they give another $\varepsilon^{\mu \rho \sigma} i p_{\sigma}$ factor. Therefore, we get a prefactor of $c /(48 \pi)$.

Matching Eq. (3.16) with Eqs. (3.18) and (3.19), we see that the MCS theory has central charge $c=$ $-\operatorname{sgn}\left(m_{t}\right)=-\operatorname{sgn}(k)$.

\section{E. Finite $T$ : Thermal Hall Effect}

We now evaluate Eq. (3.11) at nonzero T. Since we are interested in the thermal Hall effect, we will restrict ourselves to the energy-current sector $\nu=\lambda=0$.

Following some algebra using Mathematica, we find that the $(p+q)^{2}$ and $q^{2}$ factor in the denominator of $\Pi_{\mathrm{AS}}$ cancels out (we only write down the $(\mu 0 ; \rho 0)$ component here, but the cancellation happens for all components):

$$
\Pi_{\mathrm{AS}}^{\mu 0 ; \rho 0}(p)=\int \frac{\mathrm{d}^{3} q}{(2 \pi)^{3}} \frac{-m_{t} \varepsilon^{\mu \rho \sigma} u_{\sigma}(p, q)}{\left(q^{2}-m_{t}^{2}\right)\left((p+q)^{2}-m_{t}^{2}\right)},
$$

where $u_{\sigma}(p, q)$ are three polynomials in $p, q$ (superscripts denote component, not square):

$$
\begin{aligned}
& u^{0}=\frac{1}{2}\left[\left(p^{1}\right)^{2} q^{0}+p^{1} q^{1}\left(p^{0}+2 q^{0}\right)+p^{2}\left(p^{2} q^{0}+q^{2}\left(p^{0}+2 q^{0}\right)\right)\right], \\
& u^{1}=\frac{1}{2}\left[\left(p^{0}\right)^{2}\left(-q^{1}\right)+p^{0} q^{0}\left(p^{1}-2 q^{1}\right)+2\left(p^{1}\right)^{2} q^{1}+p^{2} q^{1}\left(p^{2}+2 q^{2}\right)+p^{1}\left(p^{2} q^{2}+2\left(q^{0}\right)^{2}+2\left(q^{1}\right)^{2}\right)\right], \\
& u^{2}=\frac{1}{2}\left[\left(p^{0}\right)^{2}\left(-q^{2}\right)+p^{0} q^{0}\left(p^{2}-2 q^{2}\right)+2\left(p^{2}\right)^{2} q^{2}+p^{1} q^{2}\left(p^{1}+2 q^{1}\right)+p^{2}\left(p^{1} q^{1}+2\left(q^{0}\right)^{2}+2\left(q^{2}\right)^{2}\right)\right] .
\end{aligned}
$$

To proceed, we note that $\Pi_{\mathrm{AS}}^{\mu \nu ; \rho \lambda}$ satisfies the Ward identity from both sides, so we have the ansatz

$$
\Pi_{\mathrm{AS}}^{\mu 0 ; \rho 0}(p)=m_{t} \varepsilon^{\mu \rho \sigma} p_{\sigma} A(p),
$$

and

$$
A(p)=\frac{1}{p^{2}} \int \frac{\mathrm{d}^{3} q}{(2 \pi)^{3}} \frac{-p \cdot u}{\left(q^{2}-m_{t}^{2}\right)\left((p+q)^{2}-m_{t}^{2}\right)} .
$$

We then evaluate the finite temperature part of $A(p)$, by replacing the frequency integral with a Matsubara summation

$$
\int \frac{\mathrm{d} q^{0}}{2 \pi} \rightarrow i T \sum_{q^{0}=2 \pi i T n} .
$$

The summation can be performed by standard contour methods; the finite-temperature part is

$$
\begin{aligned}
A_{\beta}(p) & =\frac{i}{p^{2}} \int \frac{\mathrm{d}^{2} \boldsymbol{q}}{(2 \pi)^{2}} \frac{1}{\left(E_{\boldsymbol{p}+\boldsymbol{q}}-E_{\boldsymbol{q}}-p^{0}\right)\left(E_{\boldsymbol{p}+\boldsymbol{q}}+E_{\boldsymbol{q}}-p^{0}\right)\left(E_{\boldsymbol{p}+\boldsymbol{q}}-E_{\boldsymbol{q}}+p^{0}\right)\left(E_{\boldsymbol{p}+\boldsymbol{q}}+E_{\boldsymbol{q}}+p^{0}\right)} \\
& \times\left\{\frac { n _ { B } ( E _ { \boldsymbol { p } + \boldsymbol { q } } ) } { E _ { \boldsymbol { p } + \boldsymbol { q } } } \left[-\boldsymbol{p}^{2} E_{\boldsymbol{p}+\boldsymbol{q}}^{4}+E_{\boldsymbol{p}+\boldsymbol{q}}^{2}\left(E_{\boldsymbol{q}}^{2} \boldsymbol{p}^{2}+\left(p^{0}\right)^{2}\left(3 \boldsymbol{p} \cdot \boldsymbol{q}+2 \boldsymbol{p}^{2}\right)-\boldsymbol{p} \cdot \boldsymbol{q}\left(\boldsymbol{p} \cdot \boldsymbol{q}+\boldsymbol{p}^{2}\right)\right)\right.\right. \\
& \left.+\left(\boldsymbol{p} \cdot \boldsymbol{q}+\boldsymbol{p}^{2}\right)\left(E_{\boldsymbol{q}}^{2}-\left(p^{0}\right)^{2}\right)\left(\left(p^{0}\right)^{2}+\boldsymbol{p} \cdot \boldsymbol{q}\right)\right] \\
& \left.-\frac{n_{B}\left(E_{\boldsymbol{q}}\right)}{E_{\boldsymbol{q}}}\left[\left(\boldsymbol{p}^{2}\left(E_{\boldsymbol{q}}^{2}+\boldsymbol{p} \cdot \boldsymbol{q}\right)+(\boldsymbol{p} \cdot \boldsymbol{q})^{2}\right)\left(-E_{\boldsymbol{p}+\boldsymbol{q}}^{2}+E_{\boldsymbol{q}}^{2}+\left(p^{0}\right)^{2}\right)+\boldsymbol{p} \cdot \boldsymbol{q}\left(p^{0}\right)^{2}\left(E_{\boldsymbol{p}+\boldsymbol{q}}^{2}+3 E_{\boldsymbol{q}}^{2}-\left(p^{0}\right)^{2}\right)\right]\right\},
\end{aligned}
$$




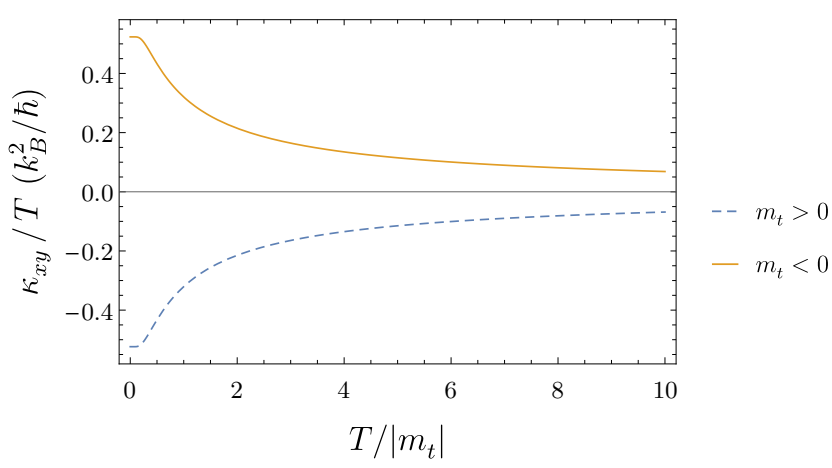

FIG. 4. The thermal Hall conductivity due to the gauge fields, from Eq. (3.29). $m_{t}$ is the topological mass.

where we have dropped the zero-temperature contribution.

To get the thermal Hall conductivity, we need to compute the Kubo conductivity $\kappa_{x y}^{\text {Kubo }}$ and the heat magnetization $M_{Q}$. As discussed earlier, because the gravitational Chern-Simons term has three derivatives, it does not contribute to $\kappa_{x y}^{\text {Kubo }}$ or $M_{Q}$, so we only need to consider the finite-temperature contributions. By inspecting (3.24), we see that $A_{\beta}\left(p^{0}, \boldsymbol{p}=0\right)=0$, and therefore, $\kappa_{x y}^{\text {Kubo }}=0$.

Sending $p^{0}, \boldsymbol{p} \rightarrow 0$ in the static limit, we get

$$
\begin{aligned}
& A\left(p^{0}=0, \boldsymbol{p} \rightarrow 0\right)= \\
& \frac{-i}{4 \pi}\left[\left|m_{t}\right| n_{B}\left(\left|m_{t}\right|\right)-T \ln \left(1-e^{-\left|m_{t}\right| / T}\right)\right] .
\end{aligned}
$$

The heat magnetization can be obtained from the differential equation

$$
\begin{aligned}
2 M_{Q}-T \frac{\partial M_{Q}}{\partial T} & =-\frac{1}{2 i} \nabla_{\boldsymbol{p}} \times \Pi_{\mathrm{AS}}^{i 0 ; 00}\left(p^{0}=0, \boldsymbol{p} \rightarrow 0\right) \\
& =-i m_{t} A\left(p^{0}=0, \boldsymbol{p} \rightarrow 0\right), \quad(3.26)
\end{aligned}
$$

where the different prefactor compared to Eq. (2.5) comes from the definition of $\Pi^{\mu \nu ; \rho \lambda}$. Integrating the above differential equation brings us to

$$
\frac{M_{Q}}{T^{2}}=C\left(m_{t}\right)-\frac{1}{4 \pi} f\left(m_{t} / T\right)
$$

where

$$
f(x)=x \ln \left(1-e^{-|x|}\right)-2 \operatorname{sgn}(x) \operatorname{Li}_{2}\left(e^{-|x|}\right),
$$

and the integration constant $C\left(m_{t}\right)$ is arbitrary function of $m_{t}$. This results in the thermal Hall conductivity

$$
\frac{\kappa_{x y}}{T}=\frac{2 M_{Q}}{T^{2}}=2 C\left(m_{t}\right)-\frac{1}{2 \pi} f\left(m_{t} / T\right),
$$

which is plotted in Fig. 4.
We choose $C\left(m_{t}\right)=-\frac{\pi}{12} \operatorname{sgn}\left(m_{t}\right)$ such that $\kappa_{x y} / T$ vanishes continuously at $m_{t}=k=0$. The physical motivations for this choice are the following. First, at $k=0$, we have the usual Maxwell theory, which should have no thermal Hall effect at any temperature. Secondly, in the high-temperature limit $T \gg m_{t}$, the system should be insensitive to the ground-state energy gap $\left(\sim m_{t}\right)$ and the related topological distinctions, so the thermal Hall coefficient should also vanish. Therefore, $\kappa_{x y} / T$ should disappear continuously at $m_{t}=k=0$ and we fix $C\left(m_{t}\right)$ accordingly.

In the $m_{t} \rightarrow \infty$ limit, we obtain

$$
\kappa_{x y} / T=-\frac{\pi}{6} \operatorname{sgn}\left(m_{t}\right)=-\frac{\pi}{6} \operatorname{sgn}(k),
$$

which, once again, yields central charge $c=$ $-\operatorname{sgn}\left(m_{t}\right)=-\operatorname{sgn}(k)$.

\section{DIRAC CHERN-SIMONS THEORY}

This section turns to a discussion of the thermal Hall response of the $\mathrm{SU}(2)$ Dirac Chern-Simons theory in Eq. (1.1) in the $1 / N_{f}$ expansion. In order to sidestep subtle issues with gravitational anomalies, we will view Eq. (1.1) as an effective theory, in which the ChernSimons term is obtained by integrating out spectator heavy Dirac fermions. To obtain a SU(2) Chern-Simons term at level $k$, we need $2|k|$ flavors of heavy Dirac fermions with mass $M$ obeying $\operatorname{sgn}(M)=\operatorname{sgn}(k)$. We will always assume $|M| \gg T$, while the ratio of the light Dirac fermion to temperature, $m / T$, can be arbitrary.

At leading order for large $N_{f}$, we ignore gauge fluctuations, and simply add the contributions of the light and heavy Dirac fermions, using the results in Section II. Owing to the $\mathrm{SU}(2)$ gauge index carried by the $\Psi$ fermions in Eq. (1.1), we need to multiply the contribution by an additional factor of 2 for each flavor. In this manner, we obtain the thermal Hall conductivity

$$
\kappa_{x y}=2 N_{f} \kappa_{x y, D}(m)+4|k| \kappa_{x y, D}(M)
$$

where $\kappa_{x y, D}(m)$ is the Dirac fermion contribution in Eq. (2.11). We show a plot of Eq. (4.1) in Fig. 5 for $N_{f}=2|k|=1$. In the limit $|M| / T \rightarrow \infty$ and $|m| / T \rightarrow$ $\infty$, Eq. (4.1) yields the first term in the square brackets in Eq. (1.3).

Upon examining the effect of gauge fluctuations in the $1 / N_{f}$ expansion, we find that there are Feynman graphs which potentially contribute to the thermal Hall conductivity even at $N_{f}=\infty$. However, evaluation of these graphs shows that they vanish, as we will illustrate in Section IV A, so no corrections are needed to Eq. (4.1) at this order. We then discuss the leading $1 / N_{f}$ corrections in Section IV B. 


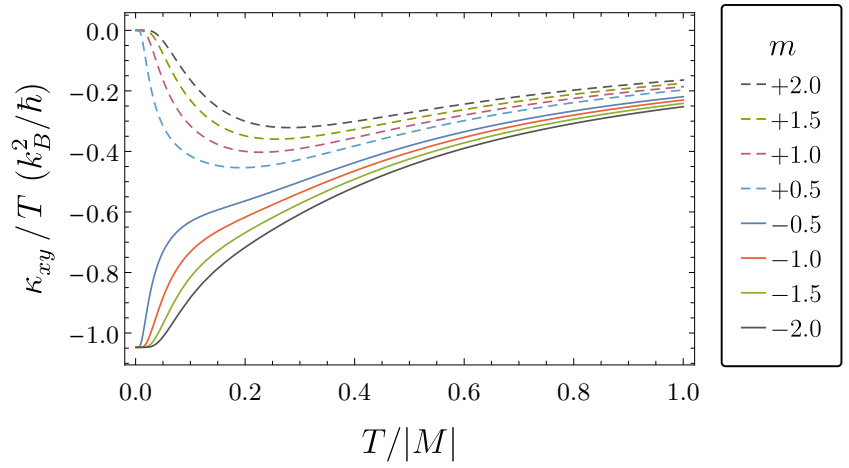

FIG. 5. The thermal hall conductivity computed from Eq. (4.1) upon including both light and heavy fermions of masses $m$ and $M=-10$, respectively for $N_{f}=2|k|=1$. The quantized value in the topological phase at zero temperature is $\pi / 3$, as expected from mean-field theory. The temperature dependence of $\kappa_{x y} / T$ calculated in this continuum field theory is in excellent agreement with the results on the lattice model in Ref. 10

\section{A. Gauge fluctuations in the $N_{f} \rightarrow \infty$ limit}

Explicitly expanding out the non-Abelian gauge field, the appropriate modification of the Lagrangian (1.1), for a particular fermion species, reads as

$$
\begin{aligned}
\mathcal{L}_{N_{f}, S U(2)} & =\sum_{v=1}^{N_{f}} \sum_{b, s, t} i \bar{\Psi}_{v s} \gamma^{\mu}\left(\partial_{\mu}-\frac{i}{\sqrt{N_{f}}} a_{\mu}^{b} \tau_{s t}^{b}\right) \Psi_{v t} \\
& +m \bar{\Psi}_{v s} \Psi_{v s},
\end{aligned}
$$

where $\tau^{a}$ are the generators of $\mathrm{SU}(2), A_{\mu}=a_{\mu}^{b} \tau^{b}$, and the coupling constant has been scaled by $1 / \sqrt{N_{f}}$ for normalization. The fermionic field is labeled simultaneously by the flavor index $v=1, \ldots, N_{f}$ as well as the color index $i=1,2,3$; the $\gamma$ (or $\sigma$ ) and $\tau$ Pauli matrices operate in Dirac and color spaces, respectively. The fermion-gluon vertex corresponds to

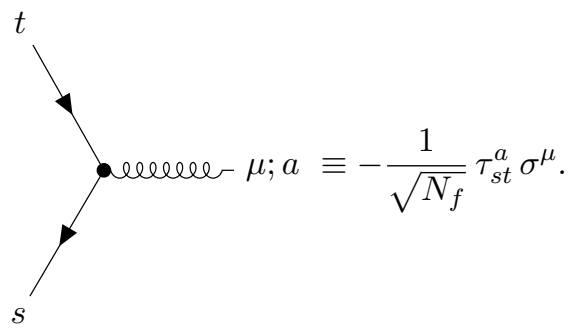

Every fermion loop now bears an extra factor of $N_{f}$ owing to the summation over flavors, while each interaction vertex carries a factor of $1 / \sqrt{N_{f}}$. The diagram in Fig. 1 is, therefore, of $\mathcal{O}\left(N_{f}\right)$. In the limit of large $N_{f} \rightarrow \infty$, the only diagram that contributes at the same order is shown in Fig. 6.

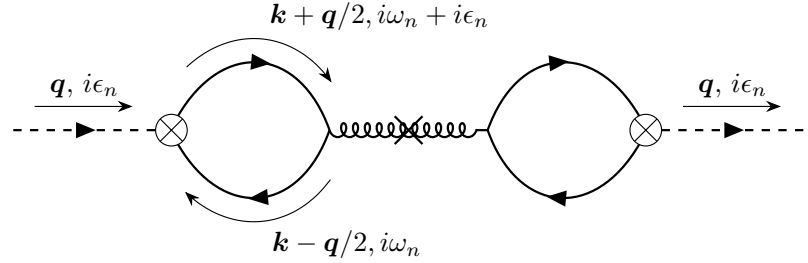

FIG. 6. The sole Feynman diagram contributing at leading order to gauge-field corrections in the limit $N_{f} \rightarrow \infty$. The dressed gluon propagator, indicated by a cross, is obtained by perturbatively integrating out the fermions from the action $\mathcal{S}$.

One might naively think that there are additional diagrams beyond Fig. 6 because each new fermion bubble within the gluon propagator is of $\mathcal{O}(1)$ in this expansion. Typically, these contributions can be subsumed in a renormalized propagator, denoted by a cross, by summing up the chain of bubble diagrams in a geometric series as

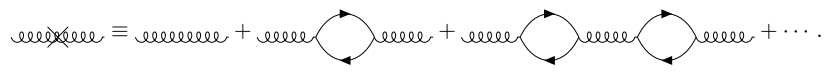

However, in $2+1 \mathrm{D}$ the Maxwell kinetic term is irrelevant and the bare $\left(F_{\mu \nu}^{a}\right)^{2}$ is thus suppressed by higher energy scales. Indeed, in our formulation, the bare kinetic term comes from integrating out the heavy fermions, and is proportional to $1 / M$. The renormalized gluon propagator should also include the contribution from integrating out light fermions, and in fact, it is dominated by the light fermion bubble. To derive the renormalized gluon propagator, we build upon the results of Ref. 36 for the photon propagator at nonzero temperatures in $2+1 \mathrm{D}$ $\mathrm{U}(1)$ gauge theories with fermionic and bosonic matter. The full expression for the gluon propagator is detailed in Appendix C.

It is now easy to observe the absence of gauge-field corrections at leading order. Figure 6 is composed of two fermion bubbles, each of which, following the Feynman rules listed earlier, translate to

$$
\begin{aligned}
& \frac{T}{V} \sum_{\boldsymbol{k}, i \omega_{n}, s t}\left(\frac{-\tau_{s t}^{a}}{\sqrt{N_{f}}}\right) \delta^{s t}\left(i \omega_{n}+i \epsilon_{n} / 2\right) \\
& \operatorname{Tr}\left[\mathcal{G}\left(\boldsymbol{k}+\boldsymbol{q} / 2, i \omega_{n}+i \epsilon_{n}\right) \sigma^{\nu} \mathcal{G}\left(\boldsymbol{k}-\boldsymbol{q} / 2, i \omega_{n}\right) \sigma^{\mu}\right],
\end{aligned}
$$

where the factor of $\delta^{s t}$ comes from the fact that the thermal vertex conserves color. Resultantly, Eq. (4.5) is just proportional to $\operatorname{Tr}\left(\tau^{a}\right)$ and hence, is identically zero. By the same reasoning, the diagram for the magnetization contribution in Fig. 7 also vanishes. Therefore, we conclude that upon taking $N_{f} \rightarrow \infty$, there are 


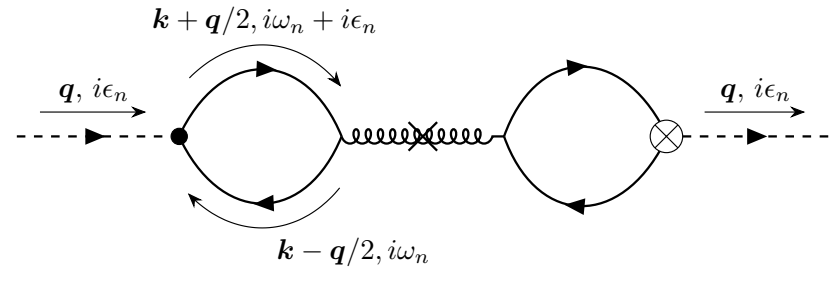

FIG. 7. The magnetization diagram at the same order in $1 / N_{f}$ as Fig. 6. One of the two thermal vertices in Fig. 6 is replaced by an energy-density vertex here.

no corrections to the thermal Hall conductivity due to $\mathrm{SU}(2)$ gauge-field fluctuations.

\section{B. Gauge fluctuations at next-to-leading order}

We are now positioned to consider the contributions to $\kappa_{x y}$ of the theory $(1.1)$ at order $N_{f}^{0}$. The Feynman diagrams which contribute to this order are shown in Figs. 8 and 9. Given the complexity of the gluon propagator in Appendix $\mathrm{C}$, and the differential equation that has to be solved for the magnetization subtraction, we do not attempt a full numerical evaluation of these graphs for general $m / T$. Instead, we will be satisfied by examining them in the limit $|m| / T \rightarrow \infty$ (recall that we always take the limit $|M| / T \rightarrow \infty)$. In this limit, we expect that a description in terms of the effective MaxwellChern-Simons theory in Section III applies, and we can therefore deduce the contribution to $\kappa_{x y}$ from results therein. The MCS theory gives the second term in the bracket of Eq. (1.3).

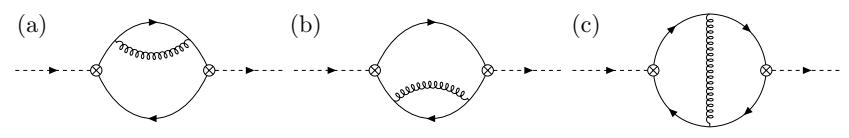

FIG. 8. The (a-b) density of states (DOS) and (c) MakiThompson [37, 38] diagrams, which contribute to $\kappa_{x y}$ for the theory (1.1) at $\mathcal{O}\left(N_{f}^{0}\right)$. Additionally, the magnetization subtraction requires evaluation of the analogous graphs given by replacing a thermal vertex with an energy-density vertex, like in Fig. 7.

We now argue that the DOS and MT diagrams listed in Fig. 8 are not important in the $|m| / T \rightarrow \infty$ limit. The DOS diagram is simply adding self-energy into the fermion propagator. A standard computation yields a fermion mass correction $\delta m \propto e^{2} / N_{f}$ in the zeromomentum limit, where $e^{2}$ is the coupling constant of the gauge field. The DOS diagram can also generate fermion anomalous dimension at higher momentum. The MT diagram is a vertex correction to the stress tensor. Using a gravitational Ward identity in [39], it can be shown that the anomalous dimension from the vertex correction cancels that from the self energy, and the net effect is a fermion mass renormalization $\delta m$ consistent with the self-energy calculation. We note in passing that similar behaviors have been observed in nonrelativistic calculations [40]. As a result, a finite renormalization $\delta m$ (also subleading in large $N_{f}$ ) can be ignored in the $|m| / T \rightarrow \infty$ limit.

Therefore, the important Feynman diagram in this limit is the 'Aslamazov-Larkin' diagram [41] drawn in Fig. 9. The triangular vertices in Fig. 9 each reduce to the stress-energy vertex used in Section III, as we now show.

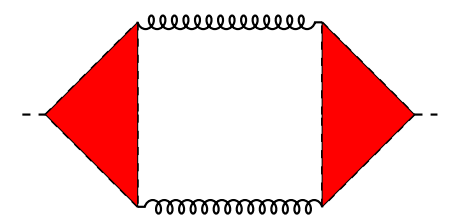

FIG. 9. The 'Aslamazov-Larkin' diagram responsible for the thermal Hall response at order $N_{f}^{0}$. The red triangles denote the effective stress tensor-gauge field-gauge field vertex obtained from integrating out fermionic loops in Fig. 10.

Following the discussions of Appendix B, the gaugeinvariant stress tensor of the theory (4.2) is

$$
\begin{aligned}
T^{\mu \nu} & =\frac{i}{2} \bar{\Psi} \gamma^{\mu}\left(\vec{\partial}^{\nu}-\overleftarrow{\partial} \nu\right) \Psi+\frac{a^{\nu b}}{\sqrt{N_{f}}} \bar{\Psi} \gamma^{\mu} \tau^{b} \Psi \\
& -\eta^{\mu \nu} \mathcal{L}_{N_{f}, S U(2)} .
\end{aligned}
$$

For shorter notation, we have suppressed flavor and color indices on the fermions.

Based on the above stress tensor, there are two types of vertices contributing to the triangular vertex, as shown in Fig. 10.
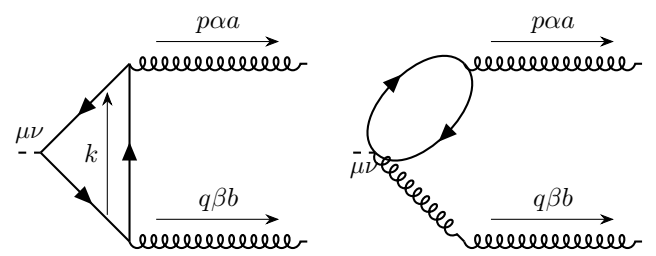

FIG. 10. The two types of diagrams for the triangular vertex. 
The first type is a fermion triangle; the corresponding effective vertex function is

$$
\begin{aligned}
\Gamma_{1}^{\mu \nu \alpha \beta}(p, q) \frac{\delta^{a b}}{2} & =\int \frac{\mathrm{d}^{3} k}{(2 \pi)^{3}}(-1) \operatorname{Tr}\left\{\left[\gamma^{\mu} \frac{(2 k+q-p)^{\nu}}{2}-\eta^{\mu \nu}\left(\frac{2 \not k+\not q-\not p}{2}+m\right)\right]\right. \\
& \left.\times \frac{i}{\not k-\not p+m} i \gamma^{\alpha} \tau^{a} \frac{i}{\not k+m} i \gamma^{\beta} \tau^{b} \frac{i}{\not k+\not q+m}\right\}+(p \alpha a \leftrightarrow q \beta b) .
\end{aligned}
$$

The second type is a fermion bubble, and the associated effective vertex is

$$
\begin{aligned}
\Gamma_{2}^{\mu \nu \alpha \beta}(p, q) \frac{\delta^{a b}}{2} & =\int \frac{\mathrm{d}^{3} k}{(2 \pi)^{3}}(-1) \operatorname{Tr}\left\{\tau^{b}\left(\gamma^{\mu} \eta^{\nu \beta}-\gamma^{\beta} \eta^{\mu \nu}\right) \frac{i}{\not k+m} i \gamma^{\alpha} \tau^{a} \frac{i}{\not k+\not p+m}\right\} \\
& +(p \alpha a \leftrightarrow q \beta b) .
\end{aligned}
$$

In the equations above, we have factored out the color indices on the LHS. Since we are looking at the $|m| / T \rightarrow$ $\infty$ limit, we will only evaluate the above integrals at zero temperature. The integrals can be performed with the standard Feynman parameter tricks. While it is possible to obtain closed-form results for arbitrary momenta and mass, the resultant expressions are too long and not very enlightening. We expand the result to second order in momenta, and obtain

$$
\Gamma_{1}^{\mu \nu \alpha \beta}(p, q)+\Gamma_{2}^{\mu \nu \alpha \beta}(p, q)=\frac{1}{12 \pi|m|} \Gamma^{\mu \nu \alpha \beta}(p, q),
$$

where $\Gamma^{\mu \nu \alpha \beta}(p, q)$ is the stress-tensor vertex function defined in (3.8).

Given the identity of the stress tensor above, we can now use the results of Section III on the Maxwell-ChernSimons theory to deduce the $1 / N_{f}$ correction to the Dirac Chern-Simons theory in the limit $|m| / T \rightarrow \infty$. Each U(1) gauge field yields the contribution in (3.30); for a $\mathrm{SU}(2)$ gauge field, we have $3 \mathrm{U}(1)$ gauge fields (which can be treated as independent at this order in $1 / N_{f}$ ), so we obtain the second term in (1.3) in the limit of large $|\hat{k}|$.

\section{CONCLUSION}

We have examined the thermal Hall conductivity in square-lattice insulators near the quantum phase transition between the Néel state and a state with coexisting Néel and semion topological order. This transition is described by the Dirac Chern-Simons field theory in (1.1) for $N_{f}=1$ and $k=-1 / 2$. The thermal Hall conductivity is expected to obey the universal scaling form in (1.2). In the limit of low $T$ away from the critical point, $|m| / T \rightarrow \infty$, we have the exact result in (1.3) obtained via a sophisticated mapping to conformal field theories on the boundary of the sample. We obtained the leading and next-to-leading order results of (1.3) in a direct $1 / N_{f}$ expansion (with $k$ taken of order $N_{f}$ ). These computations can also be applied to other values of $m / T$, and results to leading order are in (2.11) and Fig. 5; however, the next-to-leading order computations are numerically demanding.

One of the lessons of this computation is that the leading contribution can be viewed as that of fermionic matter, while the next-to-leading order terms arises from the quantum fluctuations of the gauge fields (here we are viewing the Chern-Simons term in the field theory as arising from integrating out a massive fermionic matter field).

We applied this lesson to a model of the doped antiferromagnet described by (1.6). This theory contains fermionic matter forming pocket Fermi surfaces: the thermal Hall contribution of these pockets is assumed to obey the Wiedemann-Franz law. The contribution of the gauge field was deduced from the Maxwell-ChernSimons effective action in (1.8), which has the thermal Hall contribution specified by (3.29). Importantly, this contribution has the opposite sign from the WiedemannFranz contribution, consistent with the experimental trends [2]. We note that the Dirac fermion thermal Hall conductivity in (2.11) and the Maxwell-Chern-Simons thermal Hall conductivity in (3.29) correspond to distinct universal scaling functions, a consequence of the nontopological nature of the thermal Hall effect in the quantum-critical crossover regime.

The gauge-field contribution has the correct sign to account for the additional negative contribution to $\kappa_{x y} / T$ in the pseudogap regime, as observed in Ref. 2. However, its magnitude is bounded by $\pi / 6$ [see (3.29)] for the case a single $U(1)$ gauge field. The observed magnitude is larger by, at least, a factor of 2 ; the coupling constants in (1.8) only appear in the crossover energy scale $m_{t}=\sigma_{x y} /\left(e^{2} K_{2}\right)$, and not in the overall magnitude of $\kappa_{x y}$. It is possible that other models of the pseudogap with additional gauge fields could account for the discrepancy. Alternatively, the phonon 
contribution [42] needs to be combined with the emergent gauge field to understand the observations, and a phonon-emergent photon coupling could provide the needed chirality in the phonon transport.

\section{ACKNOWLEDGMENTS}

This research was supported by the National Science Foundation under Grant No. DMR-1664842. We thank G. Grissonnanche, A. Kapustin, and L. Taillefer for helpful discussions. RS acknowledges useful discussions with Holmfridur Hannesdottir and Harley Scammell.

\section{Appendix A: Low-energy field theory}

The form of the continuum field theory is clear: upon inspection of the spectrum of the lattice model in Ref. 10 close to the phase transition, we observe two Dirac cones (of complex fermions) that result from the two sublattices of our ansatz. Therefore, the $(2 \times 2)$ Dirac matrices, $\gamma_{\mu}$, will act in sublattice space. From the lattice theory, with spinon operators $f_{i \sigma}, \sigma=\uparrow, \downarrow$, we know that there will be an SU(2) gauge field. The lattice gauge transformations act locally in the lattice model,

$$
\mathrm{SU}(2)_{g}:\left(\begin{array}{c}
f_{i \downarrow}^{\dagger} \\
f_{i \uparrow}
\end{array}\right) \longrightarrow U_{g}(i)\left(\begin{array}{c}
f_{i \downarrow}^{\dagger} \\
f_{i \uparrow}
\end{array}\right), \quad U_{g}(i) \in \mathrm{SU}(2) ;
$$

as such, they cannot mix different sublattices in the continuum model. It will therefore have the Lagrangian

$$
\mathcal{L}_{D}=i \bar{\Psi} \gamma^{\mu}\left(\partial_{\mu}-i A_{\mu}\right) \Psi+m \bar{\Psi} \Psi-\frac{1}{2} C S\left[A_{\mu}\right]
$$

with $\bar{\Psi}=\Psi^{\dagger} \gamma^{0}$. As usual, the Dirac matrices satisfy the Clifford algebra, $\left\{\gamma^{\mu}, \gamma^{\nu}\right\}=2 \eta^{\mu \nu}$ with $\eta=$ $\operatorname{diag}(+,-,-)$. The Chern-Simons term is an obvious consequence of the additional massive fermions and will be omitted in the following. To set up the notation, let us write Eq. (A2) more explicitly,

$$
\begin{gathered}
\mathcal{L}_{D}=i \Psi_{s \alpha}^{\dagger}\left(\gamma^{0} \gamma^{\mu}\right)_{s s^{\prime}}\left[\partial_{\mu}-i\left(A_{\mu}\right)_{\alpha \alpha^{\prime}}\right] \Psi_{s^{\prime} \alpha^{\prime}} \\
+m \Psi_{s \alpha}^{\dagger}\left(\gamma_{0}\right)_{s s^{\prime}} \Psi_{s^{\prime} \alpha}
\end{gathered}
$$

with sublattice and gauge index $s$ and $\alpha$, respectively. Here, the gauge transformations act as

$$
\begin{aligned}
\mathrm{SU}(2)_{g}: \quad \Psi_{s \alpha} & \rightarrow\left(U_{g}\right)_{\alpha \alpha^{\prime}} \Psi_{s \alpha^{\prime}} \\
A_{\mu} & \rightarrow U_{g} A_{\mu} U_{g}^{\dagger}-i\left(\partial_{\mu} U_{g}\right) U_{g}^{\dagger} .
\end{aligned}
$$

In the remainder of this section, we will derive Eq. (A3) from the lattice model and, thereby, relate $\Psi$ explicitly to the lattice fermions. We note that the lattice model also contains monopole operators, but we assume that these are irrelevant at the critical point [43].

For simplicity, let us focus on the case without a Zeeman field, $\boldsymbol{B}_{Z}=0$, and use the same gauge as in Ref. 10 . We define the Fourier transform as

$$
\begin{aligned}
f_{i \sigma} & =\frac{1}{\sqrt{N}} \sum_{\boldsymbol{k}} e^{i \boldsymbol{k} \boldsymbol{x}_{i}} f_{\boldsymbol{k} \sigma s(i)}, \quad \boldsymbol{x}_{i}=\left(i_{x}, i_{y}\right), \\
s(i) & = \begin{cases}A, & i_{x}+i_{y} \text { even, } \\
B, & i_{x}+i_{y} \text { odd } .\end{cases}
\end{aligned}
$$

The spectrum of the lattice model has minima at $\boldsymbol{Q}$ and $-\boldsymbol{Q}$, where $\boldsymbol{Q}=(\pi / 2,0)^{T}$, with spin polarization $\uparrow$ and $\downarrow$, respectively. Let us expand around these minima by defining new "slow", low-energy fields

$$
c_{\boldsymbol{q}, s, v=+}:=f_{\boldsymbol{Q}+\boldsymbol{q}, \uparrow, s}, c_{\boldsymbol{q}, s, v=-}:=f_{-\boldsymbol{Q}+\boldsymbol{q}, \downarrow, s},|\boldsymbol{q}| \ll 1 / a,
$$

for each of the two "valleys" $v= \pm$ and sublattices $s=$ $A, B$. Equivalently, this corresponds to

$$
f_{i \uparrow} \sim e^{i \boldsymbol{Q} \boldsymbol{x}_{\boldsymbol{i}}} c_{s(i)+}\left(\boldsymbol{x}_{i}\right), f_{i \downarrow} \sim e^{-i \boldsymbol{Q} \boldsymbol{x}_{\boldsymbol{i}}} c_{s(i)-}\left(\boldsymbol{x}_{i}\right)
$$

in real space, i.e., $c_{s, v}(\boldsymbol{r}):=N^{-1 / 2} \sum_{\boldsymbol{q}}^{\Lambda} e^{i \boldsymbol{q} \boldsymbol{r}} c_{\boldsymbol{q}, s, v}$ with some cutoff $\Lambda \ll 1 / a$. With these definitions, the meanfield Hamiltonian can be written as

$$
\begin{aligned}
H_{\mathrm{MF}} \sim & -i v_{F} \int \mathrm{d} \boldsymbol{r} c_{s, v}^{\dagger}\left[v\left(\tau_{x}\right)_{s s^{\prime}} \partial_{x}+\left(\tau_{y}\right)_{s s^{\prime}} \partial_{y}\right] c_{s^{\prime}, v} \\
& +m \int \mathrm{d} \boldsymbol{r} c_{s, v}^{\dagger} v\left(\tau_{z}\right)_{s s^{\prime}} c_{s^{\prime}, v}
\end{aligned}
$$

at low-energies. Here $v_{F}=2 t_{1}$ (which we will set to 1 in the following) and $m=-\left(4 t_{2}+N_{z} / 2\right)$, where, as in Ref. $10, t_{1}, t_{2}$, and $N_{z}$ are the nearest-, nextnearest-neighbor hopping, and the Néel order parameter, respectively; furthermore, $\tau_{j}$ denote Pauli matrices in sublattice space.

As follows from comparison of Eqs. (A1) and (A7), gauge transformation act as

$$
\mathrm{SU}(2)_{g}: C_{s}(\boldsymbol{r}) \rightarrow U_{g}^{(s)}(\boldsymbol{r}) C_{s}(\boldsymbol{r}), C_{s}(\boldsymbol{r}):=\left(\begin{array}{c}
c_{s,-}^{\dagger}(\boldsymbol{r}) \\
c_{s,+}(\boldsymbol{r})
\end{array}\right)
$$

in the low-energy theory. Naively, one might think that the gauge transformations in the two sublattices are independent as they were in the lattice model. However, this is would enhance the gauge symmetry to $\mathrm{SU}(2) \times$ $\mathrm{SU}(2)$ in the continuum model which is not the case; the reason is that not all gauge transformations allowed on the lattice act entirely in the low-energy field theory. In fact, we will see that $U_{g}^{A}(\boldsymbol{r})$ and $U_{g}^{B}(\boldsymbol{r})$ are related by a similarity transformation within the continuum field theory,

$$
U_{g}^{A}(\boldsymbol{r})=V^{\dagger} U_{g}^{B}(\boldsymbol{r}) V, \quad V \in \mathrm{SU}(2)
$$


This means that there is a gauge, reached by performing a gauge transformation with $U_{g}(i)=V^{\dagger}$ for $i_{x}+i_{y}$ even and $U_{g}(i)=\mathbb{1}$ for $i_{x}+i_{y}$ odd, where the gauge transformation is independent of $s$ in the continuum model. To see this, just note that the new field after the gauge transformation,

$$
\widetilde{C}_{s}=V_{s} C_{s}, \quad V^{A}=V^{\dagger}, \quad V^{B}=\mathbb{1},
$$

transforms as $\widetilde{C}_{s}(\boldsymbol{r}) \rightarrow V_{s}^{\dagger} U_{g}^{(s)}(\boldsymbol{r}) V_{s} \widetilde{C}_{s}(\boldsymbol{r})$; with the above choice for $V_{s}$, it holds that $V_{s}^{\dagger} U_{g}^{(s)}(\boldsymbol{r}) V_{s}=U_{g}^{B}(\boldsymbol{r})$, independent of $s$.

To add gauge-field fluctuations to the mean-field Hamiltonian (A8), we rewrite the latter in terms of the Nambu field $C_{s}$. Denoting Pauli matrices in $\mathrm{Nambu} /$ valley space by $\eta_{j}$, we get

$$
\begin{aligned}
H_{\mathrm{MF}} & \sim i \int \mathrm{d} \boldsymbol{r} C_{s}^{\dagger}\left[\left(\tau_{x}\right)_{s s^{\prime}} \partial_{x}+\left(\tau_{y}\right)_{s s^{\prime}} \partial_{y}\right] \eta_{z} C_{s^{\prime}} \\
& +m \int \mathrm{d} \boldsymbol{r} C_{s}^{\dagger}\left(\tau_{z}\right)_{s s^{\prime}} \eta_{0} C_{s^{\prime}}
\end{aligned}
$$

To bring the theory to the form of Eq. (A3), we have to transform $\eta_{z}$ into $\eta_{0}$ in the first term. This can be done by introduction of a new field $\widetilde{C}_{s}$ as defined in Eq. (A11) with $V=-i \eta_{3}$; we get

$$
\begin{aligned}
H_{\mathrm{MF}} & \sim i \int \mathrm{d} \boldsymbol{r} \widetilde{C}_{s}^{\dagger}\left[\left(\tau_{y}\right)_{s s^{\prime}} \partial_{x}-\left(\tau_{x}\right)_{s s^{\prime}} \partial_{y}\right] \eta_{0} \widetilde{C}_{s^{\prime}} \\
& +m \int \mathrm{d} \boldsymbol{r} \widetilde{C}_{s}^{\dagger}\left(\tau_{z}\right)_{s s^{\prime}} \eta_{0} \widetilde{C}_{s^{\prime}}
\end{aligned}
$$

In this form, it becomes apparent that only gauge transformations, $\widetilde{C}_{s}(\boldsymbol{r}) \rightarrow \widetilde{U}_{g}^{(s)}(\boldsymbol{r}) \widetilde{C}_{s}(\boldsymbol{r})$, that are independent of $s, \widetilde{U}_{g}^{(s)}(\boldsymbol{r})=\widetilde{U}_{g}(\boldsymbol{r})$, can appear in the low-energy theory. Based on our discussion above, we see that this indeed corresponds to Eq. (A10) with $V=-i \eta_{3}$.

To match the common conventions for the Dirac matrices,

$$
\left(\gamma^{0}, \gamma^{x}, \gamma^{y}\right)=\left(\tau_{y}, i \tau_{z}, i \tau_{x}\right)
$$

we perform yet another unitary transformation in sublattice space only (which, thus, does not affect the gauge transformation properties),

$$
\Psi_{s}(\boldsymbol{r})=\left(e^{i \frac{\pi}{4} \tau_{x}} e^{i \frac{\pi}{4} \tau_{z}}\right)_{s s^{\prime}} \widetilde{C}_{s^{\prime}}(\boldsymbol{r}),
$$

leading to

$$
\begin{aligned}
H_{\mathrm{MF}} & \sim i \int \mathrm{d} \boldsymbol{r} \Psi_{s}^{\dagger}\left[\left(\tau_{x}\right)_{s s^{\prime}} \partial_{x}-\left(\tau_{z}\right)_{s s^{\prime}} \partial_{y}\right] \Psi_{s^{\prime}} \\
& +m \int \mathrm{d} \boldsymbol{r} \Psi_{s}^{\dagger}\left(\tau_{y}\right)_{s s^{\prime}} \Psi_{s^{\prime}} .
\end{aligned}
$$

As $\Psi_{s \alpha}(\boldsymbol{r}) \rightarrow\left(U_{g}(\boldsymbol{r})\right)_{\alpha \alpha^{\prime}} \Psi_{s \alpha^{\prime}}(\boldsymbol{r})$ under gauge transformations, adding gauge fluctuations to Eq. (A16) in the action formalism leads precisely to Eq. (A3). We now know the relation between the field $\Psi$ and the lattice degrees of freedom.

\section{Appendix B: Derivation of heat current and the associated Feynman rules}

Since our eventual interest lies in the (thermal) current-current correlation functions, our first step is to compute the associated heat current vertex. There are two approaches as described below.

\section{Equation-of-motion approach}

To develop the formalism, we begin with a generalized Hubbard-like model on a lattice described by the Hamiltonian

$$
\begin{aligned}
\mathcal{H} & =\sum_{i j \mu \nu} t_{i j}^{\mu \nu} \psi_{i \mu}^{\dagger} \psi_{j \nu} \equiv \sum_{i} h_{i} \\
h_{i} & =\frac{1}{2} \sum_{j \mu \nu}\left(t_{i j}^{\mu \nu} \psi_{i \mu}^{\dagger} \psi_{j \nu}+t_{j i}^{\nu \mu} \psi_{j \nu}^{\dagger} \psi_{i \mu}\right),
\end{aligned}
$$

for which we shall evaluate the thermal current using the equation-of-motion technique [44]. We emphasize that $\mathcal{H}$ and the $\psi$ fermions need not be the same as those of the lattice model in Ref. 10; in practice, we extract $\mathcal{H}$ solely from the effective theories such as Eq. (B9) below. In the equation above, $h_{i}$ stands for the local energy density; $i, j$ are the lattice sites whereas $\mu, \nu$ connote any other degrees of freedom. It readily follows [45] that

$$
\begin{aligned}
& \dot{h}_{i}=\frac{1}{2} \sum_{j \mu \nu} \\
& {\left[t_{i j}^{\mu \nu}\left(\psi_{i \mu}^{\dagger} \dot{\psi}_{j \nu}-\dot{\psi}_{i \mu}^{\dagger} \psi_{j \nu}\right)+t_{i j}^{\nu \mu}\left(\dot{\psi}_{j \nu}^{\dagger} \psi_{i \mu}-\psi_{j \nu}^{\dagger} \dot{\psi}_{i \mu}\right)\right],}
\end{aligned}
$$

where $\dot{\mathcal{O}}=i[\mathcal{H}, \mathcal{O}]$. From the continuity equation for the energy current $[26,30,46], \dot{h}_{i}+\nabla \cdot \boldsymbol{J}_{i}^{\mathrm{E}}=0$, we find

$$
\begin{aligned}
\boldsymbol{J}_{\boldsymbol{q}}^{\mathrm{E}}=\frac{1}{\sqrt{V}} \sum_{i} e^{-i \boldsymbol{q} \cdot \boldsymbol{r}_{i}} \boldsymbol{J}_{i}^{\mathrm{E}} & \\
=\frac{i}{2 \sqrt{V}} \sum_{\boldsymbol{k} \mu \nu} \frac{\partial h_{\boldsymbol{k}}^{\mu \nu}}{\partial \boldsymbol{k}} & \left(\psi_{\boldsymbol{k}-\boldsymbol{q} / 2, \mu}^{\dagger} \dot{\psi}_{\boldsymbol{k}+\boldsymbol{q} / 2, \nu}\right. \\
& \left.\quad-\dot{\psi}_{\boldsymbol{k}-\boldsymbol{q} / 2, \mu}^{\dagger} \psi_{\boldsymbol{k}+\boldsymbol{q} / 2, \nu}\right),
\end{aligned}
$$

where $h_{\boldsymbol{k}}^{\mu \nu}$ is the second-quantized Hamiltonian and we have used the approximation $h_{\boldsymbol{k}+\boldsymbol{q} / 2}-h_{\boldsymbol{k}-\boldsymbol{q} / 2} \simeq$ $\left(\partial h_{\boldsymbol{k}} / \partial \boldsymbol{k}\right) \cdot \boldsymbol{q}$, concentrating on the small $\boldsymbol{q}$ limit. Using the Heisenberg equation of motion, this simplifies to

$$
\begin{aligned}
\boldsymbol{J}_{\boldsymbol{q}}^{\mathrm{E}} & =-\frac{1}{2 \sqrt{V}} \sum_{\boldsymbol{k}, \mu \nu \rho}\left(\frac{\partial h_{\boldsymbol{k}}^{\mu \rho}}{\partial \boldsymbol{k}} h_{\boldsymbol{k}+\boldsymbol{q} / 2}^{\rho \nu}+h_{\boldsymbol{k}-\boldsymbol{q} / 2}^{\mu \rho} \frac{\partial h_{\boldsymbol{k}}^{\rho \nu}}{\partial \boldsymbol{k}}\right) \\
& \times \psi_{\boldsymbol{k}-\boldsymbol{q} / 2, \mu}^{\dagger} \psi_{\boldsymbol{k}+\boldsymbol{q} / 2, \nu} .
\end{aligned}
$$


In this notation, it is clear that the indices $\mu, \nu$ keep track of the component of the Dirac fermion under consideration. The heat current $\left(\boldsymbol{J}^{\mathrm{Q}}\right)$ is related to the energy current by $\boldsymbol{J}^{\mathrm{Q}}=\boldsymbol{J}^{\mathrm{E}}-\mu \boldsymbol{J}$. Switching to frequency domain from Eq. (B3), at $\mu=0$, we obtain,

$$
\begin{aligned}
\boldsymbol{J}^{\mathrm{Q}}\left(\boldsymbol{q}, i \epsilon_{n}\right) & =\frac{1}{\beta \sqrt{V}} \sum_{\boldsymbol{k}, i \omega_{n}}\left(\partial_{\boldsymbol{k}} h_{\boldsymbol{k}}^{\mu \nu}\right)\left(i \omega_{n}+i \epsilon_{n} / 2\right) \\
& \times \psi_{\boldsymbol{k}-\boldsymbol{q} / 2, \mu}^{\dagger}\left(i \omega_{n}\right) \psi_{\boldsymbol{k}+\boldsymbol{q} / 2, \nu}\left(i \omega_{n}+i \epsilon_{n}\right),
\end{aligned}
$$

where $\omega_{n}$ and $\epsilon_{n}$ are fermionic and bosonic Matsubara frequencies, respectively. This defines the heat/energycurrent vertex

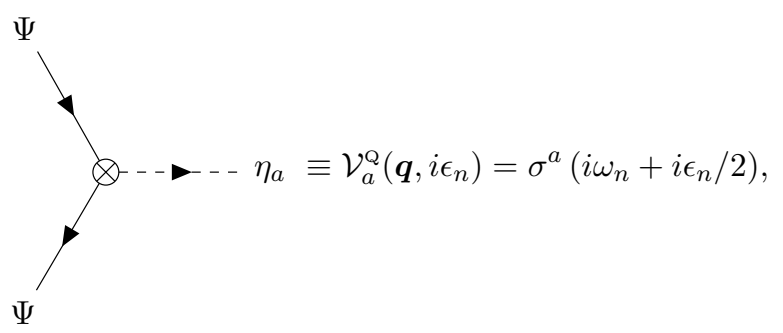

supplemented with a factor of $1 /(\beta V)$ for each internal three-momentum. Likewise, recognizing that

$$
\begin{aligned}
h(\boldsymbol{q}) & =\frac{1}{\sqrt{V}} e^{-i \boldsymbol{q} \cdot \boldsymbol{r}_{i}} h_{i} \\
& =\frac{1}{2 \sqrt{V}} \sum_{\boldsymbol{k}, \mu \nu}\left(h_{\boldsymbol{k}+\boldsymbol{q}}^{\mu \nu} \psi_{\boldsymbol{k}, \mu}^{\dagger} \psi_{\boldsymbol{k}+\boldsymbol{q}, \nu}+h_{\boldsymbol{k}}^{\nu \mu} \psi_{\boldsymbol{k}, \nu}^{\dagger} \psi_{\boldsymbol{k}+\boldsymbol{q}, \mu}\right)
\end{aligned}
$$

we have, for $\hat{K}_{\boldsymbol{q}}$ as defined in Eq. (2.5), the second vertex:

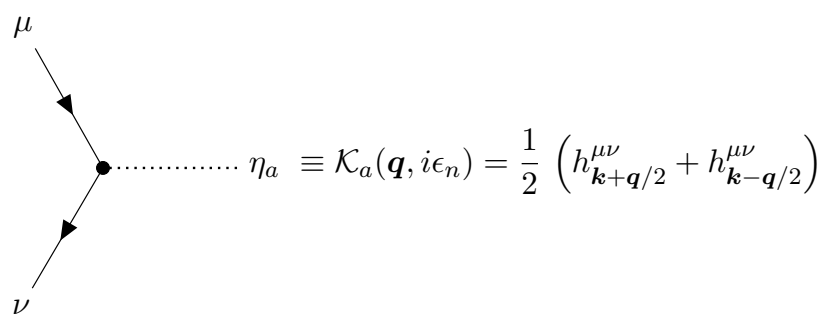

On top, for each independent momentum, a factor $1 /(\beta V)$ remains from the corresponding Fourier transform.

Lastly, since there is no specific advantage in using the conventional relativistically invariant notation of Eq. (1.1) at $T \neq 0$, in some convenient situation, we use the following equivalent form of the fermion action

$$
\begin{aligned}
\mathcal{L} & =\Psi_{\alpha}^{\dagger}\left(\partial_{\tau}-i A_{\tau}-i \boldsymbol{\sigma} \cdot(\nabla-i \boldsymbol{A})\right) \Psi_{\alpha} \\
& +m \Psi_{\alpha}^{\dagger} \sigma^{z} \Psi_{\alpha} .
\end{aligned}
$$

Then, it is not difficult to see that propagator for the fermions is simply

$$
\longrightarrow \equiv G_{\Psi}\left(\boldsymbol{k}, i \omega_{n}\right)=\frac{1}{-i \omega_{n}+\boldsymbol{\sigma} \cdot \boldsymbol{k}+m \sigma^{z}}
$$

The action and propagator for the light and heavy fermions are exactly analogous, up to an appropriate substitution of $m_{\ell}$ or $M$ for $m$ in Eq. (B10).

\section{Noether procedure}

Since, most of the time, we are dealing with a continuum field theory, it is also beneficial to directly write down the energy current or stress tensor of the field theory. In this section, we will use the Noether procedure to derive the stress tensor.

First, we shall point out that our situation is different from the standard relativistic field theory because the spacetime is not Lorentzian. For example, the DiracChern-Simons theory (1.1) comes from the underlying lattice model in Appendix. A, and the gamma matrices in (1.1) acts on band index instead of physical spin index. Therefore, under spacetime rotation the fermions transform as spinless fields, and we have explicitly broken spin-statistics relation. The consequence of nonLorentzian spacetime is that it is not always possible to covariantly couple the theory to conventional Riemannian metric and the stress tensor does not have to be symmetric. While it is possible to couple the theory to Newton-Cartan or Bargmann spacetime [47], we shall derive the stress-tensor in a simpler approach by using the Noether procedure.

Next, we review the conventional Noether procedure for the stress tensor. We first apply a gauged spacetime translation $x^{\mu} \rightarrow x^{\mu}+\varepsilon^{\mu}(x)$ to the system. Because the system is translation invariant, the leading order response to $\varepsilon$ should be $\partial \varepsilon$, and the coefficient is defined to be the stress tensor:

$$
\delta S=-\int \mathrm{d}^{3} x T_{\nu}^{\mu} \partial_{\mu} \varepsilon^{\nu}
$$

If we assume all fields transform as $\phi_{a}(x) \rightarrow \phi_{a}^{\prime}(x)=$ $\phi_{a}(x)-\varepsilon^{\mu} \partial_{\mu} \phi_{a}(x)$, we get

$$
\left(T_{\nu}^{\mu}\right)_{\text {incor. }}=\frac{\partial \mathcal{L}}{\partial\left(\partial_{\mu} \phi_{a}\right)} \partial_{\nu} \phi_{a}-\delta_{\nu}^{\mu} \mathcal{L}
$$

Here $\phi_{a}$ denotes all the field contents of the theory.

The above formalism needs further improvement, because it does not respect gauge invariance, as can be seen by applying (B12) to a Maxwell theory. The conventional Belinfante improvement (see, for example, [48]) is not applicable because it requires Lorentz 
symmetry. The spacetime-independent improvement is pointed out in [49], by requiring the gauge field to transform correctly as a one-form:

$$
\delta a_{\mu}^{a}=-\varepsilon^{\nu} \partial_{\nu} a_{\mu}^{a}-\partial_{\mu} \varepsilon^{\nu} a_{\nu}^{a} .
$$

The additional term only depends on $\partial \varepsilon$, so it does not modify the global symmetry.

The improved stress tensor is therefore

$$
T_{\nu}^{\mu}=\left(T_{\nu}^{\mu}\right)_{\text {incor. }}+\left(\frac{\partial \mathcal{L}}{\partial a_{\mu}^{a}}-\partial_{\alpha} \frac{\partial \mathcal{L}}{\partial\left(\partial_{\alpha} a_{\mu}^{a}\right)}\right) a_{\nu}^{a} .
$$

Applying the above formalism to the Dirac-ChernSimons theoy (1.1), we get

$$
T_{\nu}^{\mu}=\frac{1}{2} \bar{\Psi}_{l} i \gamma^{\mu}\left(\vec{\partial}_{\nu}-\overleftarrow{\partial}_{\nu}\right) \Psi_{l}+\bar{\Psi}_{l} \gamma^{\mu} a_{\nu} \bar{\Psi}_{l}-\delta_{\nu}^{\mu} \mathcal{L}_{\Psi}
$$

For the Maxwell-Chern-Simons theory (1.8), with $K_{1}$ and $K_{2}$ being constant, we get

$$
T^{\mu \nu}=-\bar{f}^{\mu \rho} f_{\rho}^{\nu}+\frac{1}{4} \eta^{\mu \nu} \bar{f}_{\alpha \beta} f^{\alpha \beta},
$$

where $\bar{f}_{0 i}=-\bar{f}_{i 0}=K_{2} f_{0 i}$ and $\bar{f}_{i j}=-\bar{f}_{j i}=K_{1} f_{i j}$. For the special case $K_{1}=K_{2}=1 / g$, we get back to the standard result

$$
T^{\mu \nu}=\frac{-1}{g}\left[f^{\mu \rho} f_{\rho}^{\nu}-\frac{1}{4} \eta^{\mu \nu} f_{\alpha \beta} f^{\alpha \beta}\right] .
$$

\section{Appendix C: Gluon propagator}

The first component required to stitch together the diagram 6 is the gauge boson propagator. In this subsection we extend the calculations of Ref. 36 to the case of massive fermions interacting with a $\mathrm{SU}(2)$ gauge field. The general structure of the gluon's effective action at large- $N$ follows from the Ward identity and is given by

$$
\begin{aligned}
\mathcal{S}_{A}= & \frac{T}{2} \sum_{\epsilon_{n}} \int \frac{\mathrm{d}^{2} \boldsymbol{q}}{4 \pi^{2}}\left[\left(q_{i} A_{\tau}-\epsilon_{n} A_{i}\right)^{2} \frac{D_{1}\left(\boldsymbol{q}, \epsilon_{n}\right)}{\boldsymbol{q}^{2}}\right. \\
& \left.+A_{i} A_{j}\left(\delta_{i j}-\frac{q_{i} q_{j}}{\boldsymbol{q}^{2}}\right) D_{2}\left(\boldsymbol{q}, \epsilon_{n}\right)\right]
\end{aligned}
$$

where $D_{1}$ and $D_{2}$ are functions that can be evaluated at large- $N$ by perturbatively integrating out both the fermions starting from the action $\mathcal{S}$ in Eq. (B9). In Coulomb gauge $q_{i} A_{i}=0$, this yields the nonzero elements of the propagator to be

$D_{00}^{a b}\left(\boldsymbol{q}, \epsilon_{n}\right)=\frac{4 \delta^{a b}}{D_{1}\left(\boldsymbol{q}, \epsilon_{n}\right)}$,
$D_{i j}^{a b}\left(\boldsymbol{q}, \epsilon_{n}\right)=\left(\delta_{i j}-\frac{q_{i} q_{j}}{\boldsymbol{q}^{2}}\right) \frac{4 \delta^{a b}}{D_{2}\left(\boldsymbol{q}, \epsilon_{n}\right)+\left(\epsilon_{n}^{2} / \boldsymbol{q}^{2}\right) D_{1}\left(\boldsymbol{q}, \epsilon_{n}\right)}$, where $i, j$ run over the spatial indices only. The matrix structure in color space comes from inverting the product of Pauli matrices associated with the fermion loops in the functions $D_{1,2}$ as $\sum_{s t} \tau_{s t}^{a} \tau_{t s}^{b}=\operatorname{Tr}\left(\tau^{a} \tau^{b}\right)=\delta^{a b} / 4$. No fermions remain in the action $\mathcal{S}_{A}$ and all their effects are encapsulated in Eq. (C1) through these two functions alone.

Let us begin by calculating $D_{1}$, which is defined as:

$$
\begin{aligned}
& D_{1}\left(\boldsymbol{q}, \epsilon_{n}\right)=-N \int_{\boldsymbol{k}, \omega_{n}} \operatorname{Tr}\left[\mathcal{G}\left(\boldsymbol{k}, \omega_{n}\right) \mathcal{G}\left(\boldsymbol{q}+\boldsymbol{k}, \omega_{n}+\epsilon_{n}\right)\right] \\
& =2 N \int_{\boldsymbol{k}, \omega_{n}} \frac{\omega_{n}\left(\omega_{n}+\epsilon_{n}\right)-m^{2}-\boldsymbol{k} \cdot(\boldsymbol{k}+\boldsymbol{q})}{\left(\omega_{n}^{2}+\boldsymbol{k}^{2}+m^{2}\right)\left(\left(\omega_{n}+\epsilon_{n}\right)^{2}+(\boldsymbol{k}+\boldsymbol{q})^{2}+m^{2}\right)},
\end{aligned}
$$

where we use the shorthand $\int_{\boldsymbol{k}, \omega_{n}}=T \sum_{\omega_{n}} \int \mathrm{d}^{2} \boldsymbol{k} /\left(4 \pi^{2}\right)$ to signify a summation on the internal frequencies and momenta. Using the Passarino-Veltman reduction formula [50], this can be manipulated into

$$
\begin{aligned}
& D_{1}\left(\boldsymbol{q}, \epsilon_{n}\right)=N \int_{\boldsymbol{k}, \omega_{n}}\left[\frac{-2}{\omega_{n}^{2}+\boldsymbol{k}^{2}+m^{2}}\right. \\
& \left.+\frac{\left(2 \omega_{n}+\epsilon_{n}\right)^{2}+\boldsymbol{q}^{2}}{\left(\omega_{n}^{2}+\boldsymbol{k}^{2}+m^{2}\right)\left(\left(\omega_{n}+\epsilon_{n}\right)^{2}+(\boldsymbol{k}+\boldsymbol{q})^{2}+m^{2}\right)}\right] .
\end{aligned}
$$

For the first of the two integrals here, the UV divergence is linear, so it is most convenient to use $\zeta$-function regularization [51] in which

$$
\int_{0}^{\infty} \mathrm{d} x=0, \quad \int_{1}^{\infty} \frac{1}{x} \mathrm{~d} x=\text { arbitrary. }
$$

Then, within this scheme,

$$
\begin{aligned}
& N \int_{\boldsymbol{k}, \omega_{n}} \frac{-2}{\omega_{n}^{2}+\boldsymbol{k}^{2}+m^{2}} \\
& =-N \int \frac{\mathrm{d}^{2} \boldsymbol{k}}{4 \pi^{2}} \frac{\tanh \left(\frac{1}{2} \beta \sqrt{\boldsymbol{k}^{2}+m^{2}}\right)}{\sqrt{\boldsymbol{k}^{2}+m^{2}}} \\
& =\frac{N}{2 \pi} \int \mathrm{d} k\left(1-\frac{k \tanh \left(\frac{1}{2} \beta \sqrt{k^{2}+m^{2}}\right)}{\sqrt{k^{2}+m^{2}}}\right) \\
& =\frac{N T}{\pi}\left[\ln 2+\ln \left(\cosh \left(\frac{\beta|m|}{2}\right)\right)\right] .
\end{aligned}
$$

The second integral in Eq. (C4) can be evaluated by introducing Feynman parameters and shifting the loop momentum $\boldsymbol{k} \rightarrow \boldsymbol{k}-u \boldsymbol{q}$ : 


$$
\begin{aligned}
& N \int_{0}^{1} \mathrm{~d} u \int_{\boldsymbol{k}, \omega_{n}} \frac{\left(2 \omega_{n}+\epsilon_{n}\right)^{2}+\boldsymbol{q}^{2}}{\left[u\left(\omega_{n}+\epsilon_{n}\right)^{2}+(1-u) \omega_{n}^{2}+(\boldsymbol{k}+u \boldsymbol{q})^{2}+u(1-u) \boldsymbol{q}^{2}+m^{2}\right]^{2}} \\
= & \frac{N T}{4 \pi} \sum_{\omega_{n}}\left[\left(2 \omega_{n}+\epsilon_{n}\right)^{2}+\boldsymbol{q}^{2}\right] I_{n}^{(0)} ; \text { with } I_{n}^{(0)}=\int_{0}^{1} \mathrm{~d} u \frac{1}{u\left(\omega_{n}+\epsilon_{n}\right)^{2}+(1-u) \omega_{n}^{2}+u(1-u) \boldsymbol{q}^{2}+m^{2}} \\
= & \frac{N T}{4 \pi} \sum_{\omega_{n}}\left\{\left(\left(2 \omega_{n}+\epsilon_{n}\right)^{2}+\boldsymbol{q}^{2}\right) \frac{1}{\mathcal{A}_{n}} \ln \left(\frac{2 m^{2}+\boldsymbol{q}^{2}+2 \omega_{n}^{2}+\epsilon_{n}^{2}+2 \omega_{n} \epsilon_{n}+\mathcal{A}_{n}}{2 m^{2}+\boldsymbol{q}^{2}+2 \omega_{n}^{2}+\epsilon_{n}^{2}+2 \omega_{n} \epsilon_{n}-\mathcal{A}_{n}}\right)\right\},
\end{aligned}
$$

where $\mathcal{A}_{n} \equiv \sqrt{4 m^{2} \boldsymbol{q}^{2}+\left(\boldsymbol{q}^{2}+\epsilon_{n}^{2}\right)\left(\boldsymbol{q}^{2}+\left(2 \omega_{n}+\epsilon_{n}\right)^{2}\right)}$. We shall encounter the integral $I_{n}^{(0)}$ in multiple contexts later so it is handy to define it separately. The summation over the fermionic Matsubara frequencies can only be performed numerically, and in this regard, it is useful to establish the large- $\omega_{n}$ behavior of the terms since $\omega_{n}$ is not bounded above. After symmetrizing over positive and negative frequencies and subtracting the divergent piece using a $\zeta$ regulator, the $1 / \omega_{n}$ expansion for the terms within the curly braces in Eq. (C8) stands as

$$
\begin{aligned}
& \frac{-12 m^{2}+\boldsymbol{q}^{2}+\epsilon_{n}^{2}}{3 \omega_{n}^{2}}+\frac{120 m^{4}+10 m^{2}\left(\boldsymbol{q}^{2}-15 \epsilon_{n}^{2}\right)-\boldsymbol{q}^{4}+8 \boldsymbol{q}^{2} \epsilon_{n}^{2}+9 \epsilon_{n}^{4}}{30 \omega_{n}^{4}} \\
& +\frac{1}{\omega_{n}^{6}}\left[\frac{1}{210}\left(\boldsymbol{q}^{2}+\epsilon_{n}^{2}\right)\left(\boldsymbol{q}^{4}-19 \boldsymbol{q}^{2} \epsilon_{n}^{2}+50 \epsilon_{n}^{4}\right)-4 m^{6}-m^{4}\left(\boldsymbol{q}^{2}-15 \epsilon_{n}^{2}\right)-\frac{1}{15} m^{2}\left(\boldsymbol{q}^{4}-14 \boldsymbol{q}^{2} \epsilon_{n}^{2}+105 \epsilon_{n}^{4}\right)\right]
\end{aligned}
$$

followed by terms of $\mathcal{O}\left(1 / \omega_{n}^{8}\right)$. Note that Eq. (C9) reduces correctly to the expressions documented in Ref. [36] in the limit $m \rightarrow 0$. This asymptotic behavior can then be summed by using the identities

$\sum_{n=M+1}^{\infty} \frac{1}{(2 n-1)^{2}}=\frac{1}{4 M}-\frac{1}{48 M^{3}}+\frac{7}{960 M^{5}}+\ldots$

$$
\begin{aligned}
& \sum_{n=M+1}^{\infty} \frac{1}{(2 n-1)^{4}}=\frac{1}{48 M^{3}}-\frac{1}{96 M^{5}}+\ldots, \\
& \sum_{n=M+1}^{\infty} \frac{1}{(2 n-1)^{6}}=\frac{1}{320 M^{5}}+\ldots,
\end{aligned}
$$

while retaining the exact functional dependence of Eq. (C8) for small $\omega_{n}$ up to $2 \pi( \pm M+1 / 2) T$.

We can employ a similar procedure for the second function in Eq. (C3):

$$
\begin{aligned}
D_{2}\left(\boldsymbol{q}, \epsilon_{n}\right) & =-\frac{\boldsymbol{q}^{2}}{q_{x} q_{y}} N \int_{\boldsymbol{k}, \omega_{n}} \operatorname{Tr}\left[\sigma^{x} \mathcal{G}\left(\boldsymbol{k}, \omega_{n}\right) \sigma^{y} \mathcal{G}\left(\boldsymbol{q}+\boldsymbol{k}, \omega_{n}+\epsilon_{n}\right)\right] \\
& =-\frac{2 \boldsymbol{q}^{2}}{q_{x} q_{y}} N \int_{\boldsymbol{k}, \omega_{n}} \frac{2 k_{x} k_{y}+k_{x} q_{y}+k_{y} q_{x}+m \epsilon_{n}}{\left(\omega_{n}^{2}+\boldsymbol{k}^{2}+m^{2}\right)\left(\left(\omega_{n}+\epsilon_{n}\right)^{2}+(\boldsymbol{k}+\boldsymbol{q})^{2}+m^{2}\right)} \\
& =\frac{N T}{2 \pi} \frac{\boldsymbol{q}^{2}}{q_{x} q_{y}} \sum_{\omega_{n}} \int_{0}^{1} \mathrm{~d} u \frac{2 q_{x} q_{y} u(1-u)-m \epsilon_{n}}{u\left(\omega_{n}+\epsilon_{n}\right)^{2}+(1-u) \omega_{n}^{2}+u(1-u) \boldsymbol{q}^{2}+m^{2}} \\
& =\frac{N T}{2 \pi} \frac{\boldsymbol{q}^{2}}{q_{x} q_{y}} \sum_{\omega_{n}}\left[2 q_{x} q_{y} I_{n}^{(2)}-m \epsilon_{n} I_{n}^{(0)}\right],
\end{aligned}
$$

where $I_{n}^{(0)}$ has already been calculated earlier and $I_{n}^{(2)}$ is defined as the integral

$$
I_{n}^{(2)}=\int_{0}^{1} \mathrm{~d} u \frac{u(1-u)}{u\left(\omega_{n}+\epsilon_{n}\right)^{2}+(1-u) \omega_{n}^{2}+u(1-u) \boldsymbol{q}^{2}+m^{2}}
$$




$$
\begin{aligned}
=[ & \left(2 \boldsymbol{q}^{2}\left(m^{2}+\omega_{n}^{2}\right)+\epsilon_{n}\left(2 \omega_{n}+\epsilon_{n}\right)\left(\boldsymbol{q}^{2}+2 \epsilon_{n} \omega_{n}+\epsilon_{n}^{2}\right)\right) \ln \left(\frac{\left(\mathcal{C}_{n}-\boldsymbol{q}^{2}\right)^{2}-\left(\epsilon_{n}^{2}+2 \omega_{n} \epsilon_{n}\right)^{2}}{\left(\mathcal{C}_{n}+\boldsymbol{q}^{2}\right)^{2}-\left(\epsilon_{n}^{2}+2 \omega_{n} \epsilon_{n}\right)^{2}}\right) \\
& \left.+\mathcal{C}_{n}\left(\epsilon_{n}\left(2 \omega_{n}+\epsilon_{n}\right) \ln \left(\frac{m^{2}+\left(\omega_{n}+\epsilon_{n}\right)^{2}}{m^{2}+\omega_{n}^{2}}\right)+2 \boldsymbol{q}^{2}\right)\right] \frac{1}{2 \mathcal{C}_{n} \boldsymbol{q}^{4}}
\end{aligned}
$$

with $\mathcal{C}_{n} \equiv \sqrt{4 \boldsymbol{q}^{2}\left(m^{2}+\omega_{n}^{2}\right)+\left(\boldsymbol{q}^{2}+\epsilon_{n}\left(2 \omega_{n}+\epsilon_{n}\right)\right)^{2}}$. The full expression for Eq. (C12) is forbiddingly complex and is also not particularly insightful. Instead, akin to Eq. (C9), we can symmetrize over the frequencies and write out $D_{2}$ for large $\omega_{n}$ in a series expansion as

$$
\begin{aligned}
D_{2}\left(\boldsymbol{q}, \epsilon_{n}\right) & =\frac{N T \boldsymbol{q}^{2}}{2 \pi} \sum_{\omega_{n}}\left[\frac{q_{x} q_{y}-3 m \epsilon_{n}}{3 q_{x} q_{y} \omega_{n}^{2}}+\frac{5 m \epsilon_{n}\left(6 m^{2}+\boldsymbol{q}^{2}\right)-2 q_{x} q_{y}\left(5 m^{2}+\boldsymbol{q}^{2}\right)-25 m \epsilon_{n}^{3}+7 q_{x} q_{y} \epsilon_{n}^{2}}{30 q_{x} q_{y} \omega_{n}^{4}}\right. \\
& +\frac{1}{210 q_{x} q_{y} \omega_{n}^{6}}\left\{\left(210 m^{5} \epsilon_{n}-70 m^{4} q_{x} q_{y}+70 m^{3} \epsilon_{n}\left(\boldsymbol{q}^{2}-9 \epsilon_{n}^{2}\right)-14 m^{2} q_{x} q_{y}\left(2 \boldsymbol{q}^{2}-13 \epsilon_{n}^{2}\right)\right.\right. \\
& \left.\left.\left.+7 m \epsilon_{n}\left(-13 \epsilon_{n}^{2} \boldsymbol{q}^{2}+\boldsymbol{q}^{4}+16 \epsilon_{n}^{4}\right)+q_{x} q_{y}\left(34 \epsilon_{n}^{2} \boldsymbol{q}^{2}-3 \boldsymbol{q}^{4}-13 \epsilon_{n}^{4}\right)\right)\right\}+\mathcal{O}\left(\frac{1}{\omega_{n}^{8}}\right)\right]
\end{aligned}
$$

which is convergent at large $\omega_{n}$. Once again, when $m=0$, this correctly reproduces the results of Kaul and Sachdev [36].

\section{Limit of zero external momentum}

While the propagator derived above holds for all momenta, the $\boldsymbol{q}=0$ limit, in particular, involves some subtleties and must be dealt with care. In the limit where the external momentum is zero, $D_{1}$ is finite, and according to Eq. (C8), goes to

$$
\frac{N T}{4 \pi} \sum_{\omega_{n}} \frac{\left|2 \omega_{n}+\epsilon_{n}\right|}{\left|\epsilon_{n}\right|} \ln \left(\frac{2 m^{2}+\omega_{n}^{2}+\left(\epsilon_{n}+\omega_{n}\right)^{2}+\left|\epsilon_{n}\right|\left|2 \omega_{n}+\epsilon_{n}\right|}{2 m^{2}+\omega_{n}^{2}+\left(\epsilon_{n}+\omega_{n}\right)^{2}-\left|\epsilon_{n}\right|\left|2 \omega_{n}+\epsilon_{n}\right|}\right),
$$

so the temporal component of the gluon propagator, $D_{00}^{a b}$, is nonzero. The more nontrivial part is the spatial component

$$
D_{i j}\left(\boldsymbol{q}, \epsilon_{n}\right)=\frac{\boldsymbol{q}^{2} \delta_{i j}-q_{i} q_{j}}{\boldsymbol{q}^{2} D_{2}\left(\boldsymbol{q}, \epsilon_{n}\right)+\epsilon_{n}^{2} D_{1}\left(\boldsymbol{q}, \epsilon_{n}\right)},
$$

and specifically, the behavior of $D_{2}\left(\boldsymbol{q}, \epsilon_{n}\right)$ as $\boldsymbol{q} \rightarrow 0$ :

$$
\begin{aligned}
\lim _{\boldsymbol{q} \rightarrow 0} \boldsymbol{q}^{2} D_{2}\left(\boldsymbol{q}, \epsilon_{n}\right) & =\frac{N T}{2 \pi} \sum_{\omega_{n}}\left[\epsilon_{n}\left(2 \omega_{n}+\epsilon_{n}\right) \ln \left(\frac{m^{2}+\left(\omega_{n}+\epsilon_{n}\right)^{2}}{m^{2}+\omega_{n}^{2}}\right)\right. \\
& \left.-\frac{2 m \boldsymbol{q}^{4} \epsilon_{n}}{q_{x} q_{y}\left|\epsilon_{n}\right|\left|2 \omega_{n}+\epsilon_{n}\right|}\left\{\tanh ^{-1}\left(\frac{\epsilon_{n}^{2}+2 \omega_{n} \epsilon_{n}}{\left|\epsilon_{n}\right|\left|2 \omega_{n}+\epsilon_{n}\right|}\right)+\tanh ^{-1}\left(\frac{\epsilon_{n}^{2}-2 \omega_{n} \epsilon_{n}}{\left|\epsilon_{n}\right|\left|2 \omega_{n}+\epsilon_{n}\right|}\right)\right\}\right] .
\end{aligned}
$$

Rewriting this in polar coordinates, and assuming $\cos , \sin \theta \neq 0$, we find

$$
D_{i j}\left(q, \theta, \epsilon_{n}\right) \sim \frac{q^{2} \delta_{i j}-q^{2}\left(\cos ^{4-i-j} \theta \sin ^{i+j-2} \theta\right)}{\frac{q^{4}}{q^{2} \cos \theta \sin \theta} \chi_{0}\left(\epsilon_{n}\right)+\chi_{1}\left(\epsilon_{n}\right)}=\frac{q^{4}\left(\delta_{i j}-\cos ^{4-i-j} \theta \sin ^{i+j-2} \theta\right) \cos \theta \sin \theta}{q^{4} \chi_{0}\left(\epsilon_{n}\right)+q^{2} \cos \theta \sin \theta \chi_{1}\left(\epsilon_{n}\right)}=0
$$

where the $\chi$ are functions of $\epsilon_{n}$ alone, independent of $\boldsymbol{q}$. Thus, the spatial components of the gluon propagator are zero when the external momentum is zero. The same result can be proved even when the assumption above is relaxed by successively taking the limits $q_{x} \rightarrow 0, q_{y} \rightarrow 0$. 


\section{Appendix D: Framing anomaly in the 'wrong' metric}

In the main text, we have calculated the framing anomaly using a metric compatible with the speed of 'light' $c_{0}=\sqrt{K_{1} / K_{2}}$. It would also be interesting to put the theory in an incompatible metric whose speed of 'light' is different from $c_{0}$ and redo the computation. We expect the result to be essentially the same as the one obtained from a compatible metric.

Let us assume we are in a spacetime with metric $\eta_{\mu \nu}=$ $(1,-1,-1)$, and the MCS theory (1.8) has a speed of 'light' $c_{0}=\sqrt{K_{1} / K_{2}} \neq 1$.

In momentum space, the MCS theory has the following form (we have included a gauge-fixing term)

$$
\begin{aligned}
& S=\int \frac{\mathrm{d}^{3} p}{(2 \pi)^{3}} \frac{K_{2}}{2} a_{\mu}(-p) a_{\nu}(p) \\
& \times\left(c_{0}^{2} P_{1}^{\mu \nu}(p)+P_{2}^{\mu \nu}(p)+\frac{p^{\mu} p^{\nu}}{\xi}+m_{t} \varepsilon^{\mu \nu \rho} i p_{\rho}\right) .
\end{aligned}
$$

Here $P_{1}, P_{2}$ are the transverse projectors corresponding to $\boldsymbol{B}^{2}, \boldsymbol{E}^{2}$ respectively:

$$
\begin{aligned}
& P_{1}^{\mu \nu}=\left(\begin{array}{cc}
0 & 0 \\
0 & p^{i} p^{j}-\delta^{i j} \boldsymbol{p}^{2}
\end{array}\right), \\
& P_{2}^{\mu \nu}=\left(\begin{array}{cc}
\boldsymbol{p}^{2} & p^{0} p^{j} \\
p^{0} p^{i} & \left(p^{0}\right)^{2} \delta^{i j}
\end{array}\right) .
\end{aligned}
$$

Inverting the matrix in the parenthesis of Eq. (D1), we get the gauge field propagator (in $\xi=0$ gauge)

$$
D^{\mu \nu}(p)=A_{1} P_{1}^{\mu \nu}+A_{2} P_{2}^{\mu \nu}+A_{3} \varepsilon^{\mu \nu \rho} i p_{\rho},
$$

where

$$
\begin{aligned}
A_{1} & =\frac{1}{K_{2} p^{2}\left(\tilde{p}^{2}-m_{t}^{2}\right)} \frac{\left(2-c_{0}^{2}\right)\left(p^{0}\right)^{2}-\boldsymbol{p}^{2}}{p^{2}}, \\
A_{2} & =\frac{1}{K_{2} p^{2}\left(\tilde{p}^{2}-m_{t}^{2}\right)} \frac{\tilde{p}^{2}}{p^{2}}, \\
A_{3} & =\frac{-m_{t}}{K_{2} p^{2}\left(\tilde{p}^{2}-m_{t}^{2}\right)},
\end{aligned}
$$

and $\tilde{p}^{2}=\left(p^{0}\right)^{2}-c_{0}^{2} \boldsymbol{p}^{2}$.

Next, we discuss the stress tensor $T^{\mu \nu}$. Since $K_{1} \neq$ $K_{2}$, there is no natural way to couple the system to a background metric, so we have to use Noether's theorem to derive $T^{\mu \nu}$. To ensure gauge invariance, we use a modified Noether procedure which is described in Appendix. B. Using the transformation law (B13), we can write down the stress tensor

$$
T^{\mu \nu}=-\bar{f}^{\mu \rho} f_{\rho}^{\nu}+\frac{1}{4} \eta^{\mu \nu} \bar{f}_{\alpha \beta} f^{\alpha \beta}
$$

where $\bar{f}_{0 i}=-\bar{f}_{i 0}=K_{2} f_{0 i}$ and $\bar{f}_{i j}=-\bar{f}_{j i}=K_{1} f_{i j}$. This result agrees with the energy-momentum tensor of classical electrodynamics in a medium.

The computation of the gravitational Chern-Simons term and the thermal Hall effect can now be carried out in the same way as in the main text. In this calculation, the cancellation of the $p^{2}$ factors seen in (3.20) also happens. Therefore, the denominator of the integrand is now $\left(\tilde{p}^{2}-m_{t}^{2}\right)\left((\tilde{p}+\tilde{q})^{2}-m_{t}^{2}\right)$. At zero temperature, the momentum integral can be performed in standard ways after rescaling the zeroth component, yielding the following gravitational Chern Simons term:

$$
C S_{g}[h]=\frac{-c}{192 \pi} \int \frac{\mathrm{d}^{3} p}{(2 \pi)^{3}} h_{\mu \nu}(-p) \varepsilon^{\mu \rho \sigma}\left(i p_{\sigma}\right) \tilde{P}_{T}^{\nu \lambda} h_{\rho \lambda}(p),
$$

where $\tilde{P}_{T}$ is a transverse projector in the compatible metric:

$$
\begin{aligned}
\tilde{\eta}_{\mu \nu} & =\left(c_{0}^{2},-1,-1\right), \\
\tilde{P}_{T \mu \nu} & =\tilde{\eta}_{\mu \nu} \frac{\tilde{p}^{2}}{c_{0}^{2}}-p_{\mu} p_{\nu} .
\end{aligned}
$$

A subtlety here is that all indices are raised and lowered with the incompatible metric $\eta_{\mu \nu}=(1,-1,-1)$.

As for the thermal hall effect, we compute the antisymmetrized polarization analogous to (3.20), which now becomes

$$
\Pi_{\mathrm{AS}}^{\mu 0 ; \rho 0}=\int \frac{\mathrm{d}^{3} q}{(2 \pi)^{3}} \frac{-m_{t} \varepsilon^{\mu \rho \sigma} \tilde{u}_{\sigma}}{\left(\tilde{q}^{2}-m_{t}^{2}\right)\left((\tilde{p}+\tilde{q})^{2}-m_{t}^{2}\right)},
$$

and the $\tilde{u}_{\sigma}$ 's are related to the $u_{\sigma}$ 's in the main text by simple scaling:

$$
\begin{aligned}
& \tilde{u}^{0}\left(p^{0}, q^{0}, \boldsymbol{p}, \boldsymbol{q}\right)=c_{0}^{2} u^{0}\left(p^{0}, q^{0}, c_{0} \boldsymbol{p}, c_{0} \boldsymbol{q}\right) \\
& \tilde{u}^{i}\left(p^{0}, q^{0}, \boldsymbol{p}, \boldsymbol{q}\right)=c_{0} u^{i}\left(p^{0}, q^{0}, c_{0} \boldsymbol{p}, c_{0} \boldsymbol{q}\right) .
\end{aligned}
$$

Carrying out the integration, we found that (3.25) is not altered, and therefore the thermal Hall coefficient remains to be

$$
\kappa_{x y}=\frac{\pi}{6} c T
$$

[1] Y. Kasahara, K. Sugii, T. Ohnishi, M. Shimozawa, M. Yamashita, N. Kurita, H. Tanaka, J. Nasu, Y. Mo- tome, and T. Shibauchi, "Unusual Thermal Hall Effect in a Kitaev Spin Liquid Candidate $\alpha-\mathrm{RuCl}_{3}$," Phys. 
Rev. Lett. 120, 217205 (2018).

[2] G. Grissonnanche, A. Legros, S. Badoux, E. Lefrançois, V. Zatko, M. Lizaire, F. Laliberté, A. Gourgout, J. S. Zhou, S. Pyon, T. Takayama, H. Takagi, S. Ono, N. Doiron-Leyraud, and L. Taillefer, "Giant thermal hall conductivity in the pseudogap phase of cuprate superconductors," Nature 571, 376 (2019).

[3] R. Samajdar, S. Chatterjee, S. Sachdev, and M. S. Scheurer, "Thermal Hall effect in square-lattice spin liquids: A Schwinger boson mean-field study," Phys. Rev. B 99, 165126 (2019).

[4] J. H. Han, J.-H. Park, and P. A. Lee, "Consideration of thermal Hall effect in undoped cuprates," Phys. Rev. B 99, 205157 (2019).

[5] S. Sachdev, M. A. Metlitski, Y. Qi, and C. Xu, "Fluctuating spin density waves in metals," Phys. Rev. B 80, 155129 (2009).

[6] M. S. Scheurer, S. Chatterjee, W. Wu, M. Ferrero, A. Georges, and S. Sachdev, "Topological order in the pseudogap metal," Proc. Nat. Acad. Sci. 115, E3665 (2018).

[7] W. Wu, M. S. Scheurer, S. Chatterjee, S. Sachdev, A. Georges, and M. Ferrero, "Pseudogap and FermiSurface Topology in the Two-Dimensional Hubbard Model," Phys. Rev. X 8, 021048 (2018).

[8] S. Sachdev, "Topological order, emergent gauge fields, and Fermi surface reconstruction," Rep. Prog. Phys. 82, 014001 (2019).

[9] W. Wu, M. S. Scheurer, M. Ferrero, and A. Georges, "Not all doped Mott insulators have a pseudogap: key role of van Hove singularities," (2019), arXiv:2001.00019 [cond-mat.str-el].

[10] R. Samajdar, M. S. Scheurer, S. Chatterjee, H. Guo, C. Xu, and S. Sachdev, "Enhanced thermal Hall effect in the square-lattice Néel state," Nature Phys. 15, 1290 (2019).

[11] A. Cappelli, M. Huerta, and G. R. Zemba, "Thermal transport in chiral conformal theories and hierarchical quantum Hall states," Nucl. Phys. B636, 568 (2002).

[12] N. Seiberg, T. Senthil, C. Wang, and E. Witten, "A Duality Web in 2+1 Dimensions and Condensed Matter Physics," Ann. Phys. 374, 395 (2016).

[13] C. Closset, T. T. Dumitrescu, G. Festuccia, Z. Komargodski, and N. Seiberg, "Contact Terms, Unitarity, and F-Maximization in Three-Dimensional Superconformal Theories," J. High Energ. Phys. 10, 053 (2012).

[14] C. Closset, T. T. Dumitrescu, G. Festuccia, Z. Komargodski, and N. Seiberg, "Comments on Chern-Simons Contact Terms in Three Dimensions," JHEP 09, 091 (2012).

[15] K. Damle and S. Sachdev, "Nonzero-temperature transport near quantum critical points," Phys. Rev. B 56, 8714 (1997).

[16] S. Sachdev, "Nonzero-temperature transport near fractional quantum Hall critical points," Phys. Rev. B 57, 7157 (1998).

[17] J. He, C. R. Rotundu, M. S. Scheurer, Y. He, M. Hashimoto, K.-J. Xu, Y. Wang, E. W. Huang, T. Jia, S. Chen, B. Moritz, D. Lu, Y. S. Lee, T. P.
Devereaux, and Z.-x. Shen, "Fermi surface reconstruction in electron-doped cuprates without antiferromagnetic long-range order," Proc. Natl. Acad. Sci. 116, 3449 (2019).

[18] P. A. Lee, "Gauge field, Aharonov-Bohm flux, and high$T_{c}$ superconductivity," Phys. Rev. Lett. 63, 680 (1989).

[19] L. Balents and S. Sachdev, "Dual vortex theory of doped Mott insulators," Ann. Phys. 322, 2635 (2007).

[20] M. S. Scheurer and S. Sachdev, "Orbital currents in insulating and doped antiferromagnets," Phys. Rev. B 98, 235126 (2018).

[21] B. I. Halperin, P. A. Lee, and N. Read, "Theory of the half-filled Landau level," Phys. Rev. B 47, 7312 (1993).

[22] H. Katsura, N. Nagaosa, and P. A. Lee, "Theory of the thermal Hall effect in quantum magnets," Phys. Rev. Lett. 104, 066403 (2010).

[23] B. K. Agarwalla, L. Zhang, J.-S. Wang, and B. Li, "Phonon Hall effect in ionic crystals in the presence of static magnetic field," Eur. Phys. J. B 81, 197 (2011).

[24] R. Matsumoto and S. Murakami, "Theoretical prediction of a rotating magnon wave packet in ferromagnets," Phys. Rev. Lett. 106, 197202 (2011).

[25] L. Smrcka and P. Streda, "Transport coefficients in strong magnetic fields," J. Phys. C: Solid State Phys. 10, 2153 (1977).

[26] N. R. Cooper, B. I. Halperin, and I. M. Ruzin, "Thermoelectric response of an interacting two-dimensional electron gas in a quantizing magnetic field," Phys. Rev. B 55, 2344 (1997).

[27] T. Qin, Q. Niu, and J. Shi, "Energy Magnetization and the Thermal Hall Effect," Phys. Rev. Lett. 107, 236601 (2011).

[28] J. M. Luttinger, "Theory of thermal transport coefficients," Phys. Rev. 135, A1505 (1964).

[29] S. Ryu, J. E. Moore, and A. W. W. Ludwig, "Electromagnetic and gravitational responses and anomalies in topological insulators and superconductors," Phys. Rev. B 85, 045104 (2012).

[30] A. Kapustin and L. Spodyneiko, "Thermal hall conductance and a relative topological invariant of gapped twodimensional systems," Phys. Rev. B 101, 045137 (2020).

[31] R. Matsumoto, R. Shindou, and S. Murakami, "Thermal Hall effect of magnons in magnets with dipolar interaction," Phys. Rev. B 89, 054420 (2014).

[32] This is allowed since we focus on the limit $\boldsymbol{q}=0$. More formally, this corresponds to expanding the full integrand in powers of $q_{x}$ and $q_{y}$ and then keeping only those terms of $\mathcal{O}\left(q_{x}\right)$ that would survive the $\boldsymbol{k}$ integration.

[33] E. Witten, "Quantum field theory and the Jones polynomial," Commun. Math. Phys 121, 351 (1989).

[34] M. Asorey, F. Falceto, J. L. Lopez, and G. Luzon, "Universality and ultraviolet regularizations of ChernSimons theory," Nucl. Phys. B429, 344 (1994).

[35] M. Stone, "Gravitational anomalies and thermal Hall effect in topological insulators," Phys. Rev. B 85, 184503 (2012).

[36] R. K. Kaul and S. Sachdev, "Quantum criticality of U(1) gauge theories with fermionic and bosonic matter in two 
spatial dimensions," Phys. Rev. B 77, 155105 (2008).

[37] K. Maki, "The critical fluctuation of the order parameter in type-II superconductors," Prog. Theor. Phys 39, 897 (1968).

[38] R. S. Thompson, "Microwave, flux flow, and fluctuation resistance of dirty type-II superconductors," Phys. Rev. B 1, 327 (1970).

[39] R. Brout and F. Englert, "Gravitational ward identity and the principle of equivalence," Phys. Rev. 141, 1231 (1966).

[40] D. R. Niven and R. A. Smith, "Absence of singular superconducting fluctuation corrections to thermal conductivity," Phys. Rev. B 66, 214505 (2002).

[41] L. G. Aslamazov and A. I. Larkin, "Effect of fluctuations on the properties of a superconductor at temperatures above the critical temperature," Fiz. Tverd. Tela 10, 1104 (1968), [Sov. Phys. Solid State, 10, 875 (1968)].

[42] J.-Y. Chen, S. A. Kivelson, and X.-Q. Sun, "Enhanced thermal Hall effect in nearly ferroelectric insulators," (2019), arXiv:1910.00018 [cond-mat.str-el].

[43] X.-Y. Song, Y.-C. He, A. Vishwanath, and C. Wang, "From spinon band topology to the symmetry quantum numbers of monopoles in Dirac spin liquids," Phys. Rev. X 10, 011033 (2020), arXiv:1811.11182 [cond-mat.strel].
[44] G. D. Mahan, Many-Particle Physics, 3rd ed., Physics of Solids and Liquids (Springer Science \& Business Media, New York, 2000).

[45] I. Paul and G. Kotliar, "Thermal transport for manybody tight-binding models," Phys. Rev. B 67, 115131 (2003).

[46] K. Michaeli and A. M. Finkel'stein, "Quantum kinetic approach for studying thermal transport in the presence of electron-electron interactions and disorder," Phys. Rev. B 80, 115111 (2009).

[47] M. Geracie, K. Prabhu, and M. M. Roberts, "Physical stress, mass, and energy for non-relativistic matter," J. High Energ. Phys. 2017, 89 (2017).

[48] P. Di Francesco, P. Mathieu, and D. Sénéchal, Conformal field theory, Graduate texts in contemporary physics (Springer, New York, NY, 1997).

[49] T. Brauner, "Noether currents of locally equivalent symmetries," (2020).

[50] G. Passarino and M. Veltman, "One-loop corrections for $e^{+} e^{-}$annihilation into $\mu^{+} \mu^{-}$in the Weinberg model," Nucl. Phys. B 160, 151 (1979).

[51] A. Y. Shiekh, "Zeta-function regularization of quantum field theory," Can. J. Phys. 68, 620 (1990). 